\title{
Information Management and Pricing in Platform Markets*
}

Bruno Jullien

Toulouse School of Economics, CNRS, Toulouse
Alessandro Pavan

Northwestern University and CEPR

May 2018

\begin{abstract}
We study platform markets in which the information about users' preferences is dispersed. First, we show how the dispersion of information introduces idiosyncratic uncertainty about participation decisions and how the latter shapes the elasticity of the demands and the equilibrium prices. We then study the effects on profits, consumer surplus, and welfare of platform design, blogs, forums, conferences, advertising campaigns, post-launch disclosures, and other information management policies affecting the agents' ability to predict participation decisions on the other side of the market.
\end{abstract}

JEL classification: D82

Keywords: platform markets, dispersed information, design, information management, informative advertising, market ignition, global games

\footnotetext{
*Earlier versions circulated under the title "Platform Competition under Dispersed Information," and "Platform Pricing under Dispersed Information." For useful comments and suggestions, we thank Philipp Kircher, anonymous referees, Mark Armstrong, Emilio Calvano, Carmelo Cennamo, David Evans, Jens Förderer, Andrei Hagiu, Andrea Ordanini, Marco Ottaviani, Margherita Pagani, Emmanuela Prandelli, Benoit Reillier, Patrick Rey, Michael Riordan, Andre Veiga, Jean Tirole, Glen Weyl and seminar participants at Berkeley Haas, Bocconi University, the 2013 CSIOIDEI Conference, Universidad Carlos III of Madrid, NYU Stern, the 2013 SAET Conference, the 2013 Berlin IO Day, the 2014 Econometric Society European Meetings, and the 2015 Shanghai Microeconomics workshop. Pavan also thanks the National Science Foundation (grant SES-60031281) and Nokia for financial support and the University of California at Berkeley and Bocconi University for hospitality. Emil Temnyalov, Jonas Mishara Blomberger, Xavier Lambin, Fernando Stipanicic Marquez, and Riccardo Bianchi Vimercati provided excellent research assistance. The financial support of the European Research Council (ERC) under the European Union's Horizon 2020 research and innovation programme (grant agreement No 670494) is gratefully acknowledged.
} 


\section{Introduction}

"The sheer number and complexity of instruments being used by platform owners, including investments, technology rules, information dissemination, contracting choices, and pricing, is an empirical phenomenon deserving closer attention and clearer explanation" (Boudreau and Hagiu, (2009))

Motivation. When Apple launched its first iPhone in 2007, the market responded with hesitation: developers were uncertain about the attractiveness for end-users of the phone's touch-screen feature, while end-users were uncertain about the potential for new applications. While subsequent versions of the iPhone were received with more enthusiasm, most versions of Windows smart phones have been received with hesitations. ${ }^{1}$

The launch of a new platform (e.g., a new smart-phone operating system, or a video-games console) comes with uncertainty about the platform's ability to attract agents from various sides of the market. ${ }^{2}$ Because of such uncertainty, platforms invest considerable resources in information management policies such as advertising campaigns, exhibitions, information disclosures, community management, forums, showrooms, and blogs aimed at promoting on each side of the market the platform's ability to attract users from other sides. The importance of such policies, especially at the early stages of a platform's life, when communication and information management are key factors for igniting the market, is well recognized by practitioners and market designers. It stems from the fact that platforms recognize that potential users need to form beliefs about the participation of other sides of the market and that such beliefs are typically based not only on information that is publicly available but also on the agents' own personal experience with the platform's product and services. ${ }^{3}$ Information management and pricing thus complement each other in improving the coordination between the various sides of a market.

Perhaps surprisingly, such information management policies have received little attention in the academic literature. ${ }^{4}$ The assumption commonly made in the analysis of platform markets is that the distribution of preferences is common knowledge, so that each agent from each side can perfectly predict the participation decisions of all other agents and such predictions coincide with the platforms'. While such assumption simplifies the analysis, it does not square well with markets in which the product, or service, offered by one, or multiple, platforms is relatively new. Importantly, such a simplifying assumption precludes the analysis of how platforms invest in information management to influence the agents' beliefs and thereby their participation decisions.

\footnotetext{
${ }^{1}$ An issue faced by Microsoft in the smart-phone market has been the self-defeating nature of the agents' expectations. See, e.g., "Microsoft Banks on Mobile Apps", by Shira Ovide and Ian Sherr, April 5, 2012, The Wall-Street Journal.

${ }^{2}$ Uncertainty also plays a major role in the launch of new operating systems for personal computers. For example, many of the Microsoft OS releases were received with hesitations from various sides of the market (e.g., Windows 3, Windows 95, Windows 7).

${ }^{3}$ See, e.g., Evans and Schmalensee (2015).

${ }^{4}$ For an overview of the economics literature on platforms see, e.g., Rochet and Tirole (2006), Rysman (2009), and Weyl (2010).
} 
Contribution. In this paper, we develop a rich, yet flexible, framework that can be used to shed light on the key trade-offs that platforms face when designing their information management policies. We then use such a framework to investigate the effects of such policies on profits, consumer surplus, and welfare. We address the following questions: (a) How is pricing affected by the uncertainty that agents face about a platform's ability to attract participation from other sides? (b) What are the strategic implications of such uncertainty for the initial development of a platform, in particular for its design decisions? (c) Which communication strategies can help managing expectations and convince potential adopters of the likely take-off of a platform? In particular, which features of a platform should be prominently released? (d) Should platforms commit to disclose to late adopters about earlier participation decisions? (e) What are the effects of the above policies on consumer surplus and welfare?

To answer the above questions, first, we study how uncertainty shapes the elasticity of the demands and the equilibrium prices. We then investigate the platforms' incentives to favor design dimensions that appeal to both sides of the market (e.g., features of the operating system that are likely to be appreciated by both end-users and app developers), as well as their incentives to manage expectations through various information policies. Such policies include standard advertising campaigns but also less conventional practices such as the promotion of forums, conferences, and community architectural design that platforms use to help potential adopters predict participation decisions on other sides of the market. Finally, we investigate the platforms' incentives to disseminate information about earlier participation decisions.

Key insights. Our first result shows that, when preferences are aligned across sides, dispersed information weakens competition. This is because it reduces the elasticity of the demands on both sides of the market by introducing positive correlation between the agents' stand-alone valuations (i.e., the direct utility the agents derive from the platforms' products) ${ }^{5}$, and their estimated network effects (i.e., the indirect utility the agents expect from interacting with agents from other sides of the market). ${ }^{6}$ To illustrate, suppose that, on each side, agents with a higher stand-alone valuation for a platform's product expect, on average, a higher appreciation also by agents from the opposite side. Then suppose that a platform were to raise its price on, say, side 1. Because the marginal agent from side 1 who is excluded is the most "pessimistic" about the side-2's participation, among those who would join the platform without the price change, the drop in the side-1's demand expected by the platform is smaller than in a market where all agents share the same beliefs about the side-2's participation (as is necessarily the case under complete information). In this case, the dispersion

\footnotetext{
${ }^{5}$ This direct utility may originate from the one-sided goods, or services, that the platform bundles with its two-sided services (see e.g., Edelman (2015)), or from preassigned agents on the opposite side (e.g., vertically integrated suppliers, as in Lee (2013), or marquee buyers, as in Rochet and Tirole, (2003)). Such direct utilities are also referred to in the literature as "intrinsic benefits" (e.g., Armstrong and Wright, (2007)), or "membership benefits" (e.g., Weyl, (2010)).

${ }^{6}$ Other expressions favored in the literature are "usage values" (e.g., Rochet and Tirole, (2006)), "cross-side externalities" (e.g., Armstrong, (2006)), and "interaction benefits" (e.g., Weyl, (2010)).
} 
of information reduces the own-price elasticity of the demand functions. Other things equal, such reduced elasticity may contribute to higher prices on one side and lower prices on the opposite side. However, in general, both the sum and the skewness of the equilibrium prices across sides are higher under dispersed information than under complete information.

The above results have interesting implications for the effects on platforms' profits, consumer surplus, and welfare, of design and information management policies aimed at influencing the agents' expectations about the participation of other sides. To investigate such implications, we distinguish between pre-launch campaigns affecting the agents' expectations about the other side's participation by acting primarily on the correlation between the agents' appreciations for a platform's product and post-launch campaigns affecting the expectations of late adopters though the dissemination of information about earlier participation decisions.

In the case of pre-launch campaigns, we show that aligning preferences across sides unambiguously contributes to higher profits. In designing its features, a platform thus may gain more by focusing on a niche in which agents from both sides share similar preferences than to aim at getting on board a broader base of undifferentiated users on each side of the market. Moreover, when preferences are aligned, platforms gain from pre-launch disclosures, the promotion of showrooms, trials, and other disclosure policies that help agents use their own appreciation for a platform's products to predict participation decisions on the other side of the market. ${ }^{7}$ Unfortunately, such policies, while welfare enhancing, may hurt consumers by increasing equilibrium prices more than they increase consumers' gross surplus.

To study the effects of post-launch disclosures, we consider a dynamic extension in which agents have the possibility of changing platform over time. We study the effects of post-launch campaigns providing late adopters with information about earlier participation decisions. We show that while such campaigns have a positive effect on welfare, they have ambiguous effects on profits. On the one hand, by permitting the late adopters to learn about the true distribution of preferences through the observation of the earlier participation decisions, such campaigns reduce the sensitivity of the agents' expectations about the other side's participation to their own appreciation for the platforms' products. Because a lower sensitivity contributes to a higher price-elasticity of the demands, through this channel, such campaigns contribute to lower profits. At the same time, by improving the overall quality of the agents' information, such campaigns also permit agents from different sides to better coordinate on which platform to join. Better coordination softens ex-ante competition by contributing to a higher ex-post differentiation between platforms. Hence, through this second channel, post-launch disclosures contribute to higher profits. The net effect of such campaigns then depends on which of the above two channels prevails. When preferences are drawn from a Gaussian distribution, we show that the second channel tends to prevail, making post-launch disclosures profitable. We also show that the effects of such post-launch disclosures on profits are strongest when

\footnotetext{
${ }^{7}$ See Godes \& al. (2005) for a discussion of the role of social interactions in marketing and Haruvy and Prasad (2005) and Gaudeul (2010) for the analysis of freewares and sharewares.
} 
preferences are sufficiently aligned and network effects are large.

In the Supplementary Material, we extend the analysis in a few directions. First, we contrast a platform's incentives to engage in design and information management in a covered duopoly market to their counterparts in an uncovered monopolistic market. We find that, in general, the comparison is ambiguous. However, in markets that are symmetric across sides, the effects of such policies on monopoly profits are always smaller than in a duopoly market. In particular, in markets in which the monopolist serves at least half of the market on each side, the aforementioned pre-launch disclosures have negative effects on monopoly profits, while they have positive effects on duopoly profits. Second, we discuss the robustness of the key insights to opt-outs and multi-homing. Third, we consider a dynamic extension in which platforms change prices at the same frequency at which agents learn the relative attractiveness of the two platforms. Lastly, we consider markets in which cross-side network effects interact with within-side network effects.

Outline. The rest of the paper is organized as follows. We wrap up the Introduction with a brief discussion of the contribution of the paper to the pertinent literature. Section 2 presents the model. Section 3 introduces some benchmarks that facilitate the subsequent analysis. Section 4 characterizes demand functions and equilibrium prices under dispersed information. Section 5 contains all the implications for platform design, advertising, forums, blogs, post-launch disclosures, and other information campaigns. Section 6 offers a few concluding remarks. All proofs are either in the Appendix or in the paper's Supplementary Material.

(Most) pertinent literature. Our model is the incomplete-information analog of the models of differentiated platforms with homogenous network effects of Rochet and Tirole (2003, 2006), and Armstrong (2006). Weyl (2010) and White and Weyl (2017) extend these models focusing on the heterogeneity of network effects, respectively in a monopolistic market and in a duopoly (see also Ambrus and Argenziano, 2009, for how heterogenous network effects can lead to asymmetric networks), while Tan and Zhou (2017) extend these models by allowing for an arbitrary number of platforms and for richer specifications of the within-side and cross-side network effects. These papers, however, do not consider the possibility that preferences be correlated across sides, which is one of the key forces behind the mechanism we consider in the present paper. We thus identify a new channel by which the dispersion of information affects the elasticity of the demands on the two sides and thereby the equilibrium prices. This in turn permits us to uncover novel effects. For example, under complete information, it is the discrepancy between the importance assigned to network effects by the marginal user and by the average user that is responsible for distortions in prices and in network allocations, along the lines of those identified in Spence (1975) and Sheshinski (1976) — see Weyl (2010). In contrast, under dispersed information, it is the discrepancy between the participation rates expected by the marginal agent on each side and the participation rates expected by the platforms that is responsible for novel distortions.

Our paper focuses on dispersed information at the subscription stage pertaining to preferences 
in the population. In contrast, Halaburda and Yehezkel (2013) examine optimal tariffs when agents privately learn their valuations only after joining a platform but before transacting with the other side. Hagiu and Halaburda (2014) focus on information about prices, showing that, unlike in the monopoly case, in a duopoly market, platforms may prefer agents to remain uninformed about the prices charged on the opposite side. While these papers address very different questions, they both point to the importance of asymmetric information for platforms' pricing decisions.

The paper also contributes to the literature on informative advertising and marketing campaigns. The closest contributions are Anderson and Renault (2006, 2009) and Johnson and Myatt (2006) (see also the references therein). These papers show that, in a one-sided market, information about horizontal differences between products may reduce the intensity of competition by increasing product differentiation, an effect that is present also in our model. However, we focus on novel effects that are specific to two-sided platforms. In particular, we focus on campaigns that help agents predict other agents' preferences and behavior, which is new and brings novel implications. Johnson and Myatt (2006) also analyze product design from the perspective of dispersion of demand, showing that firms prefer extremal dispersion. Our contribution shows that this conclusion does not extend to two-sided platforms. ${ }^{8}$

Finally, the paper contributes to the literature on coordination under incomplete information, and in particular the global-games literature. ${ }^{9}$ A difference though is that, in our work, the dispersion of information is a central part of the phenomenon under examination, as opposed to a convenient tool for equilibrium selection. To the best of our knowledge, this is the first paper to examine a global game in which two distinct populations (the two sides) coordinate under dispersed information and where the outcome of such coordination is shaped by two competing "big players" (the platforms). The paper in this literature closest to ours is Argenziano (2008) which uses a global-game approach to study one-sided network competition. The questions addressed in that paper relate to allocative efficiency under product differentiation and differ from those addressed in the present paper which are largely motivated by the two-sideness of the markets under examination.

\section{Model}

Players. Two platforms, indexed by $k=A, B$, compete on two sides, $i=1,2$. Each side is populated by a measure-one continuum of agents, indexed by $l \in[0,1]$.

Actions and payoffs. Each agent $l$ from each side $i$ must choose which platform to join. The

\footnotetext{
${ }^{8}$ Veiga et al. (2017) study one-sided platform design focusing on the correlation between heterogeneous valuation for quality and heterogeneous contribution to network effects. These features are absent in our model, which focuses on consumers' information and the correlation of valuations across sides.

${ }^{9}$ See, among others, Carlsson and Van Damme (1993), and Morris and Shin (2003).
} 
payoff that each agent $l$ from each side $i$ derives from joining each platform $k$ is given by

$$
U_{i l}^{k}=v_{i l}^{k}+\gamma_{i} q_{j}^{k}-p_{i}^{k}
$$

where $v_{i l}^{k}$ is the agent's stand-alone valuation for platform $k$ (think of this as the direct utility the agent derives from the platform's product, or service), $q_{j}^{k} \in[0,1]$ is the mass of agents from side $j \neq i$ that join the platform, $\gamma_{i} \in \mathbb{R}_{+}$is a parameter that controls for the intensity of the network effects on side $i$, and $p_{i}^{k}$ is the price (the access fee) charged by the platform to the side- $i$ agents. ${ }^{10}$ As in most of the literature, we abstract from price discrimination. By paying the fee $p_{i}^{k}$, each agent $l$ from side $i$ receives the platform's product and/or is granted access to all agents joining the same platform from the opposite side of the market. The payoff that each agent $l$ from each side $i$ obtains from not joining any platform (that is, the agent's outside option) is assumed to be equal to zero.

Each platform's payoff is the total revenue collected from the two sides: ${ }^{11}$

$$
\Pi^{k}=p_{1}^{k} q_{1}^{k}+p_{2}^{k} q_{2}^{k}
$$

All players are risk-neutral expected-utility maximizers.

We focus on equilibria with full participation and single-homing. To illustrate the key ideas in the simplest possible way, we then assume that each agent's stand-alone valuations for the products of the two platforms are given by

$$
v_{i l}^{A}=s_{i}-\frac{v_{i l}}{2} \text { and } v_{i l}^{B}=s_{i}+\frac{v_{i l}}{2} \text { all } l \in[0,1], i=1,2 .
$$

This specification is chosen so that the "type" of each agent is unidimensional and coincides with the differential in stand-alone valuations $v_{i l} \equiv v_{i l}^{B}-v_{i l}^{A}, i=, 1,2$, which measures the extent to which the intrinsic characteristics of platform $B$ (i.e. set aside expected network effects) are preferred to those of platform $A$ by an agent of type $l$ in side $i$. The scalar $s_{i} \in \mathbb{R}$, which we assume to be commonly known to the platforms and to all agents, only serves the role of guaranteeing that full participation is robust to the possibility that agents opt out of the market (see the analysis in the Supplementary Material).

Illustrative example. To fix ideas, let the two platforms be two mobile software platforms, say Apple and Google, or two video-game consoles, say Nintendo Wii and Microsoft X-box. Let the side1 agents be end-user consumers, and the side- 2 agents be app, or, alternatively, game developers. Interpret the end-users' differentials in stand-alone valuations as the difference in utility derived from the two software's core functions (alternatively, from the two consoles' build-in services and

\footnotetext{
${ }^{10}$ To ease the exposition, we assume positive network effects on both sides. All the results extend to markets in which network effects are negative on one side (as in certain advertising models) provided that $\gamma_{1}+\gamma_{2}>0$, which, empirically, appears the most relevant case.

${ }^{11}$ All results extend to markets in which the platforms incur costs to get agents on-board and/or to provide the agents with the auxiliary products/services they provide. Because these costs do not play any fundamental role, we disregard them to facilitate the exposition.
} 
proprietary games). Then interpret the developers' differentials as the differences in profit from developing for one or the other platform. ${ }^{12}$ Such differences in profits in turn may originate in differences in developing costs (due to the heterogeneity in the two codes), developer support, and APIs, i.e., application programming interfaces. In this example, the price paid by the end-users to the platforms should then be interpreted as the price for purchasing the hardware (e.g., the smartphone, or the videogame console), while the price paid by the developers should be interpreted as the fee asked by the platforms to place the app in the corresponding library. ${ }^{13}$

Beliefs. The distribution of the agents' stand-alone valuations on the two sides of the market (hereafter, "the aggregate state") is unknown both to the platforms and to each agent. This is the key point of departure from the rest of the literature. We assume each agent's differential in standalone valuations $v_{i l}$ parametrizes both the agent's preferences for the products/services of the two platforms and the agent's beliefs about the aggregate state, which in turn he uses to form beliefs about the participation of the other side of the market. For simplicity, we assume that platforms do not possess private information. This assumption permits us to abstract from the signaling role of prices (and the associated multiplicity of equilibria), and focus instead on the novel effects that come from the agents' uncertainty about the participation of the other side of the market.

For any $v \in \mathbb{R}, i=1,2$, we denote by $Q_{i}^{A}(v)$ the measure of agents from side $i$ that platform $A$ expects to have a differential in stand-alone valuations no greater than $v$, and by $Q_{i}^{B}(v)$ the measure of agents from side $i$ that platform $B$ expects to have a differential in stand-alone valuations no smaller than $v$. As it will become clear in a moment, these functions also capture the participation rates expected by the two platforms. ${ }^{14}$

We assume that each function $Q_{i}^{k}$ is strictly monotone and differentiable over the entire real line and then denote by $d Q_{i}^{k}(v) / d v$ its derivative.

Next, consider the agents. For any $i, j=1,2, j \neq i$, any $\left(v_{i}, v_{j}\right) \in \mathbb{R}^{2}$, let $M_{j i}\left(v_{j} \mid v_{i}\right)$ denote the measure of agents from side $j$ with differential in stand-alone valuations no greater than $v_{j}$, as expected by any agent from side $i$ with differential in stand-alone valuations equal to $v_{i}$. These functions are thus the cumulative distribution functions of the agents' marginal beliefs over the crosssectional distribution of differentials in stand-alone valuations on the other side of the market. In the context of the motivating example introduced above, such functions capture heterogeneity in beliefs, both among the end-users and the developers, about the platforms' ability to attract participation

\footnotetext{
${ }^{12}$ We focus here on apps, or games, developed for a single platform. The case of apps developed for multiple platforms corresponds to the case of "multihoming", which we do not develop in this paper-see, however, the discussion in the Supplement for the robustness of the main insights to multihoming.

${ }^{13}$ Apple, for example, charges currently $\$ 99$ for placing an app in its App Store, whereas Google charges $\$ 25$ to place an app in its Google Play Console.

${ }^{14}$ These functions in turn can be related to the platforms' beliefs over the distribution of valuations in the crosssection of the population. Letting $\Psi_{i}^{k}(v)$ denote the cumulative distribution function of platform $k^{\prime}$ 's marginal beliefs over the side- $i$ cross-sectional distribution of differentials in stand-alone valuations, we have that $Q_{i}^{A}(v)=\Psi_{i}^{A}(v)$ and $Q_{i}^{B}(v)=1-\Psi_{i}^{B}(v)$.
} 
from the other side of the market. For example, when applied to the consumers' side, such functions capture how end-user consumers use their own appreciation for the features of a new smartphone, or a new video-game console, to form expectations about the development of new apps. Likewise, when applied to the developers side, such functions capture how potential developers use available information about a platform's software characteristics (e.g., its interface) to predict the adoption by end-users. Importantly, such beliefs need not coincide with the platforms' own expectations.

Hereafter, we assume that, for any $v_{i}, M_{j i}\left(v_{j} \mid v_{i}\right)$ is strictly increasing in $v_{j}$, and differentiable in each argument, $i, j=1,2, j \neq i$. As for the dependence of $M_{j i}\left(v_{j} \mid v_{i}\right)$ on $v_{i}$, we will focus primarily on two polar cases.

Definition 1. Preferences are aligned if, for all $v_{j} \in \mathbb{R}, M_{j i}\left(v_{j} \mid v_{i}\right)$ is decreasing in $v_{i}, i, j=1,2$, $j \neq i$. Conversely, preferences are misaligned if, for all $v_{j} \in \mathbb{R}, M_{j i}\left(v_{j} \mid v_{i}\right)$ is increasing in $v_{i}$, $i, j=1,2, j \neq i$.

In markets in which preferences are aligned, agents with a higher appreciation for a platform's product or service also expect a higher appreciation by agents from the opposite side, whereas the opposite is true in markets in which preferences are misaligned. In the context of our motivating example, preferences are aligned when those end-users who are most enthusiastic about the new smartphone's operating system, or the new videogame console, are also those who are most optimistic about the development of new apps and games for the new platform, and likewise those developers who are most enthusiastic about the platform's characteristics are also those who are most optimistic about the platform's ability to convince end-users to buy the new smartphone or the new console.

Importantly, note that the definition does not presume that stand-alone valuations be drawn from a common prior. It simply establishes a monotone relationship between beliefs and stand-alone valuations. Finally note that, while the above specification assumes that agents face uncertainty about the aggregate distribution of stand-alone valuations for each of the two platforms' products, all the results extend to markets in which the stand-alone valuations for one of the two platforms' product (say, an incumbent's) are commonly known.

Common prior. As mentioned already, the above specification does not impose that the agents' and the platforms' beliefs be generated from a common prior. To see how the special case of a common prior fits into the framework introduced above, it is convenient to think of the following set-up. The aggregate state (that is, the cross-sectional joint distribution of stand-alone valuations on the two sides of the market) is parametrized by $\theta \in \Theta$. All players believe that $\theta$ is drawn from a distribution $F_{\theta}$. In each state $\theta$, each agent's differential in stand-alone valuations, $v_{i l}$, is drawn from a distribution $\Lambda_{i}^{\theta}$, with density $\lambda_{i}^{\theta}\left(v_{i}\right)$, independently across agents, $i=1,2, l \in[0,1]$. The mass of agents from side $i$ with valuation no greater than $v$ in state $\theta$ is then equal to $\Lambda_{i}^{\theta}\left(v_{i}\right)$. In this case, the platforms' and the agents' beliefs are given by

$$
Q_{i}^{A}\left(v_{i}\right)=\mathbb{E}_{\theta}\left[\Lambda_{i}^{\theta}\left(v_{i}\right)\right], Q_{i}^{B}\left(v_{i}\right)=\mathbb{E}_{\theta}\left[1-\Lambda_{i}^{\theta}\left(v_{i}\right)\right], \text { and } M_{j i}\left(v_{j} \mid v_{i}\right)=\frac{\mathbb{E}_{\theta}\left[\Lambda_{j}^{\theta}\left(v_{j}\right) \lambda_{i}^{\theta}\left(v_{i}\right)\right]}{\mathbb{E}_{\theta}\left[\lambda_{i}^{\theta}\left(v_{i}\right)\right]},
$$


where all expectations are computed under the common prior $F_{\theta} \cdot{ }^{15}$

\section{Timing.}

- At stage 1, platforms simultaneously set prices on each side.

- At stage 2, after observing the platforms' prices, and after observing their own stand-alone valuations, all agents simultaneously choose which platform to join.

- At stage 3, payoffs are realized.

As anticipated above, we focus on equilibria in which agents single-home and where no agent opts out of the market. In the baseline model, we thus assume that all agents must join one of the two platforms and cannot join both. In the Supplementary Material, however, we identify conditions under which the equilibrium allocations in the baseline model are also equilibrium allocations in a more general game in which all agents can opt out of the market and/or multi-home.

Finally, hereafter, we say that a strategy profile for the agents constitutes a continuation (BayesNash) equilibrium in the game that starts after the platforms announce their prices if the action taken by each agent's type constitutes a best response to all other agents' strategies.

\section{Benchmarks}

We start by considering two benchmarks. The first one is a market without network effects. The second is a market with complete information. These benchmarks serve the purpose of illustrating that the novel effects we document below originate precisely in the combination of network effects with dispersed information.

Benchmark 1 (absence of network effects). Suppose that $\gamma_{1}=\gamma_{2}=0$. In this case, the demand that each platform expects on each side is independent of the price it sets on the opposite side of the market.

Given the prices $p_{i}^{A}$ and $p_{i}^{B}$ set by the two platforms on side $i=1,2$, each agent $l$ from side $i$ whose differential in stand-alone valuations $v_{i l}$ exceeds the threshold $\hat{v}_{i}=p_{i}^{B}-p_{i}^{A}$ joins platform $B$, whereas each agent with differential $v_{i l}<\hat{v}_{i}$ joins platform $A$. The (direct residual) demand curve that each platform $k$ expects on each side $i$ is thus given by $Q_{i}^{k}\left(\hat{v}_{i}\right)$. We then have the following result:

\footnotetext{
${ }^{15}$ Note that, as standard in the definition of Bayesian games, irrespective of whether or not beliefs are consistent with a common prior, the mappings $M_{j i}\left(\cdot \mid v_{i}\right)$ from types to beliefs are common knowledge. A common prior simply imposes restrictions on these mappings, as well as on the platforms' beliefs. Finally note that the functions $Q_{i}^{k}$ and $M_{j i}$ do not provide a complete description of the platforms' and of the agents' beliefs. However, as we show below, they summarize all the information that is relevant for the equilibrium prices and the agents' participation decisions.
} 
Proposition 1. Suppose there are no network effects (i.e., $\gamma_{i}=0, i=1,2$ ). The equilibrium prices, along with the participation thresholds they induce, must satisfy the following conditions

$$
p_{i}^{k}=\frac{Q_{i}^{k}\left(\hat{v}_{i}\right)}{\left|d Q_{i}^{k}\left(\hat{v}_{i}\right) / d v_{i}\right|},
$$

along with $\hat{v}_{i}=p_{i}^{B}-p_{i}^{A}, i=1,2, k=A, B$.

The conditions for the optimal prices are the familiar conditions for a duopoly equilibrium with horizontally differentiated products. Clearly, in this benchmark, the dispersion of information is irrelevant. The prices set by the two platforms reflect only the platforms' own beliefs.

Benchmark 2 (complete information). Next, suppose network effects are positive, but assume information is complete, meaning that the cross-sectional distribution of stand-alone valuations is commonly known by the platforms and by all agents. The platforms' beliefs then coincide with the beliefs of any other agent. Formally,

$$
M_{j i}\left(v_{j} \mid v_{i}\right)=Q_{j}^{A}\left(v_{j}\right)=1-Q_{j}^{B}\left(v_{j}\right), \text { all }\left(v_{i}, v_{j}\right) \in \mathbb{R}^{2}, i, j=1,2, j \neq i .
$$

In the Appendix (see Lemma 1), we show that, as long as the network effects are not too large, for any vector of prices $p \equiv\left(p_{1}^{A}, p_{2}^{A}, p_{1}^{B}, p_{2}^{B}\right)$ set by the two platforms, there exists a unique continuation equilibrium and it is in threshold strategies. ${ }^{16}$ All side- $i$ agents whose differential in stand-alone valuations exceeds $\hat{v}_{i}$ join platform $B$, whereas all agents whose differential is below $\hat{v}_{i}$ join platform $A$, where the thresholds $\hat{v}_{1}$ and $\hat{v}_{2}$ are given by the unique solution to

$$
\hat{v}_{i}=p_{i}^{B}-p_{i}^{A}+\gamma_{i}\left[Q_{j}^{A}\left(\hat{v}_{j}\right)-Q_{j}^{B}\left(\hat{v}_{j}\right)\right], i, j=1,2, j \neq i .
$$

Now fix the prices set by platform $A$. For any vector of participation rates $\left(Q_{1}, Q_{2}\right)$, the prices that platform $B$ must set to implement the participation rates $Q_{1}$ and $Q_{2}$ are given by

$$
p_{i}^{B}=p_{i}^{A}+\hat{v}_{i}+2 \gamma_{i} Q_{j}-\gamma_{i} \text {, with } \hat{v}_{i} \text { s.t. } Q_{i}^{B}\left(\hat{v}_{i}\right)=Q_{i}, i, j=1,2, j \neq i .
$$

Platform $B$ 's profits (expressed as a function of the participation rates $\left(Q_{1}, Q_{2}\right)$ ) are thus equal to

$$
\Pi^{B}=\sum_{i, j=1,2, j \neq i}\left[p_{i}^{A}+\hat{v}_{i}+2 \gamma_{i} Q_{j}-\gamma_{i}\right] Q_{i}
$$

We then have the following result:

Proposition 2. Suppose information is complete. The equilibrium prices, along with the participation thresholds they induce, must satisfy the following conditions

$$
p_{i}^{k}=\frac{Q_{i}^{k}\left(\hat{v}_{i}\right)}{\left|d Q_{i}^{k}\left(\hat{v}_{i}\right) / d v_{i}\right|}-2 \gamma_{j} Q_{j}^{k}\left(\hat{v}_{j}\right)
$$

along with $\hat{v}_{i}=p_{i}^{B}-p_{i}^{A}+\gamma_{i}\left[Q_{j}^{A}\left(\hat{v}_{j}\right)-Q_{j}^{B}\left(\hat{v}_{j}\right)\right], i, j=1,2, j \neq i$.

\footnotetext{
${ }^{16}$ For large network effects, the continuation equilibrium is not unique. See, among others, Caillaud and Jullien (2001, 2003). For small network effects, instead, the unique continuation equilibrium is also the unique rationalizable strategy profile. It thus does not require that agents be able to coordinate with other agents. This is appealing, especially in large markets, which are the focus of the paper.
} 
The profit-maximizing prices thus solve the familiar optimality conditions (5) according to which each platform's price on each side equals the platform one-sided inverse semi-elasticity, $Q_{i}^{k}\left(\hat{v}_{i}\right) /\left|d Q_{i}^{k}\left(\hat{v}_{i}\right) / d v_{i}\right|$, adjusted by the effect of a variation in side- $i$ 's participation on side- $j$ 's willingness to pay (the term $2 \gamma_{j} Q_{j}^{k}$ in (5) - see, for example, Weyl (2010)).he

\section{Pricing under Dispersed Information}

We now turn to the case where network effects interact with dispersed information.

\subsection{Preliminaries: Monopoly Analysis}

As a first step toward the characterization of the duopoly equilibrium, but also as a convenient tool to introduce some of the key ideas in the simplest possible way, suppose, for a moment, that there is a single platform in the market and, without loss of generality, let such a platform be platform $B$. Then drop all superscripts pertaining to the platform's identity to ease the notation. Importantly, the platform does not necessarily cover the entire market. In the context of the illustrative example introduced above, this means that, given the price for the platform's new smartphone (or new console), certain end-users may decide to stay with the old phone, or old video-game console. ${ }^{17}$ Likewise, given the price asked by the platform to the developers to place an app in its library, certain developers may prefer to not develop for the platform.

Recall that the payoff that each agent $l \in[0,1]$ from each side $i=1,2$ obtains from the outside option is equal to zero and that his stand-alone valuation for platform $B$ 's product is equal to $s_{i}+v_{i l} / 2$. Each agent $l \in[0,1]$ from each side $i=1,2$ then joins the platform if

$$
s_{i}+\frac{v_{i l}}{2}+\gamma_{i} \mathbb{E}\left[q_{j} \mid v_{i l}\right] \geq p_{i}
$$

and opts out if the inequality is reversed, where $\mathbb{E}\left[q_{j} \mid v_{i l}\right]$ is the side- $j$ participation expected by the agent. Provided the network effects are not too large, we then have that, for any vector of prices $\left(p_{1}, p_{2}\right)$, the demand expected by the platform on each side $i=1,2$ is given by $Q_{i}\left(\hat{v}_{i}\right)$, where $\left(\hat{v}_{1}, \hat{v}_{2}\right)$ is the unique solution to the system of equations given by ${ }^{18}$

$$
s_{i}+\frac{\hat{v}_{i}}{2}+\gamma_{i}\left[1-M_{j i}\left(\hat{v}_{j} \mid \hat{v}_{i}\right)\right]=p_{i}, i, j=1,2, j \neq i .
$$

Now, again in the context of our illustrative example, suppose the platform aims at getting on board $Q_{1}$ end-users from side one and $Q_{2}$ developers from side 2. Which prices should it set?

\footnotetext{
${ }^{17}$ Keeping the old console may be viewed as a case where those agents who do not join platform $B$ join another platform with a large base of pre-committed agents on all sides, for which the participation of additional users does not bring significant additional network effects.

${ }^{18}$ The precise bound on the network effects that guarantees uniqueness of the demand system, for any price vector, is the same as the one identified by Conditions (M) and (Q) below in the duopoly case — see the Supplementary Material (Lemma S1) for details.
} 
Obviously, because the platform does not know the exact state of the world (i.e., the exact distribution of preferences among the end-users and of the exact distribution of opportunity costs among the developers), the quantities $Q_{1}$ and $Q_{2}$ must be interpreted as the expected participation rates, where the expectation is taken over all possible states of the world using the platform's own beliefs. Given the platform's beliefs, the prices $\left(p_{1}, p_{2}\right)$ should thus be set so as to induce participation thresholds $\hat{v}_{1}$ and $\hat{v}_{2}$ satisfying $Q_{i}\left(\hat{v}_{i}\right)=Q_{i}, i=1,2$. Using (7), we then have that the prices should be set equal to

$$
p_{i}=s_{i}+\frac{\hat{v}_{i}}{2}+\gamma_{i}\left[1-M_{j i}\left(\hat{v}_{j} \mid \hat{v}_{i}\right)\right]
$$

Differences in beliefs between the platform and the agents then manifest themselves in the difference between $Q_{j}$ and $1-M_{j i}\left(\hat{v}_{j} \mid \hat{v}_{i}\right)$, where the former is the side- $j$ participation expected by the platform and the latter is the side- $j$ participation expected by the side- $i$ marginal agent (the one who is just indifferent between joining and not joining the platform). The novel effects then originate from the fact that, holding $Q_{j}$ constant, any change in the side- $i$ participation expected by the platform comes with a variation in the side- $i$ 's marginal agent's beliefs about the side- $j$ participation according to

$$
\frac{\partial \mathbb{E}\left[q_{j} \mid \hat{v}_{i}\right]}{\partial \hat{v}_{i}}=\frac{\partial\left[1-M_{j i}\left(\hat{v}_{j} \mid \hat{v}_{i}\right)\right]}{\partial \hat{v}_{i}} .
$$

When network effects are positive, as assumed here, and preferences are aligned between the two sides, this novel effect contributes to steeper inverse demand curves, whereas the opposite is true when preferences are misaligned. This is intuitive. When preferences are aligned, the new marginal agent that the platform brings on board when it lowers its price on side $i$ is less optimistic about the side- $j$ 's participation than those agents who are already on board (the infra-marginal agents). This means that, to get the new marginal agent on board, the reduction in the side- $i$ price must be larger than the one necessary to induce the same increase in the side- $i$ participation under complete information. Interestingly, this novel effect is present even if the platform adjusts its price on side $j$ so as to maintain the side- $j$ participation threshold $\hat{v}_{j}$ fixed (which amounts to maintaining the side- $j$ 's demand $Q_{j}$ expected by the platform constant).

The above novel effects play an important role for equilibrium prices, as we show next. Given the bijective relationship between the prices $\left(p_{1}, p_{2}\right)$ and the participation thresholds $\left(\hat{v}_{1}, \hat{v}_{2}\right)$ given by (8), we have that the prices $\left(p_{1}, p_{2}\right)$ maximize the platform's profits if and only if the participation thresholds $\left(\hat{v}_{1}, \hat{v}_{2}\right)$ they induce solve the following problem:

$$
\max _{\left(v_{1}, v_{2}\right)} \hat{\Pi}\left(v_{1}, v_{2}\right) \equiv \sum_{i, j=1,2, j \neq i}\left\{s_{i}+\frac{v_{i}}{2}+\gamma_{i}\left[1-M_{j i}\left(v_{j} \mid v_{i}\right)\right]\right\} Q_{i}\left(v_{i}\right) .
$$

We then have the following result:

Proposition 3. Suppose a single platform is active in the market and information is dispersed. The profit-maximizing prices, along with the participation thresholds they induce, must satisfy the 
following optimality conditions

$$
p_{i}=\frac{Q_{i}\left(\hat{v}_{i}\right)}{2\left|d Q_{i}\left(\hat{v}_{i}\right) / d v_{i}\right|}-\gamma_{j} \frac{\partial M_{i j}\left(\hat{v}_{i} \mid \hat{v}_{j}\right)}{\partial v_{i}} \frac{Q_{j}\left(\hat{v}_{j}\right)}{\left|d Q_{i}\left(\hat{v}_{i}\right) / d v_{i}\right|}-\gamma_{i} \frac{\partial M_{j i}\left(\hat{v}_{j} \mid \hat{v}_{i}\right)}{\partial v_{i}} \frac{Q_{i}\left(\hat{v}_{i}\right)}{\left|d Q_{i}\left(\hat{v}_{i}\right) / d v_{i}\right|},
$$

along with $s_{i}+\frac{\hat{v}_{i}}{2}+\gamma_{i}\left[1-M_{j i}\left(\hat{v}_{j} \mid \hat{v}_{i}\right)\right]=p_{i}, i, j=1,2, j \neq i$.

Note that the price formulas in (11) are the incomplete-information analogs of the familiar complete-information optimality conditions ${ }^{19}$

$$
P_{i}=-\frac{\partial P_{i}\left(Q_{1}, Q_{2}\right)}{\partial Q_{i}} Q_{i}-\frac{\partial P_{j}\left(Q_{1}, Q_{2}\right)}{\partial Q_{i}} Q_{j} .
$$

They require that profits do not change when the platform increases the expected side- $i$ participation and then adjusts the side- $j$ price to maintain the expected side- $j$ participation constant.

In particular, the first term in (11) is the familiar inverse semi-elasticity of the demand curve in the absence of network effects.

The second term in (11) captures the extra benefit of cutting the price on side $i$ that comes from the possibility of raising the price on side $j$ as a result of attracting more agents from side $i$ (see, e.g., Rochet and Tirole, 2006 and Armstrong, 2006). The novelty, relative to complete information, is that the variation in the side- $i$ participation that the platform expects to trigger by varying its side- $i$ price now differs from the variation expected by the side- $j$ marginal agent. This novel effect is captured by the term

$$
\left.\frac{\partial \mathbb{E}\left[q_{i} \mid \hat{v}_{j}\right]}{\partial Q_{i}}\right|_{\hat{v}_{j}=\text { const }}=\frac{\partial M_{i j}\left(\hat{v}_{i} \mid \hat{v}_{j}\right)}{\partial v_{i}} \frac{1}{\left|d Q\left(\hat{v}_{i}\right) / d v_{i}\right|}
$$

in (11), which measures the sensitivity of the beliefs of the side- $j$ 's marginal agent to changes in the demand expected by the platform on side $i{ }^{20}$ Note that, irrespective of whether preferences are aligned or misaligned between the two sides, this term is always positive, thus contributing to a lower price on side $i$.

The third term in (11) is the most interesting one, for this term is absent under complete information. As explained above, this term originates in the fact that a variation in the side- $i$ demand expected by the platform implies a variation in the side- $i$ marginal agent's beliefs about the side $j$ 's participation. When preferences are aligned, by steepening the slope of the inverse demand, this term contributes to reducing the platform's incentives to lower its side- $i$ price. The opposite is true when preferences are misaligned.

\subsection{Duopoly Analysis}

We now return to the duopoly game where both platforms are active in the market. As usual, we solve the game backwards by considering first the continuation game that starts after the platforms

\footnotetext{
${ }^{19}$ To see this, note that $\frac{\partial P_{i}}{\partial Q_{i}}=\frac{\partial P_{i} / \partial v_{i}}{\partial Q_{i} / \partial v_{i}}=\frac{1-\gamma_{i} \partial M_{j i}\left(\hat{v}_{j} \mid \hat{v}_{i}\right) / \partial v_{i}}{d Q_{i} / d v_{i}}$ and $\frac{\partial P_{j}}{\partial Q_{i}}=\frac{\partial P_{j} / \partial v_{i}}{\partial Q_{i} / \partial v_{i}}=\frac{\gamma_{j} \partial M_{i j}\left(\hat{v}_{i} \mid \hat{v}_{j}\right) / \partial v_{i}}{d Q_{i} / d v_{i}}$.

${ }^{20}$ Note that, under complete information, instead, $\partial M_{i j}\left(\hat{v}_{i} \mid \hat{v}_{j}\right) / \partial v_{i}=\left|d Q_{i}\left(\hat{v}_{i}\right) / d v_{i}\right|$, in which case the second term in (11) reduces to $-\gamma_{j} Q_{j}\left(\hat{v}_{j}\right)$.
} 
set their prices $p=\left(p_{1}^{A}, p_{2}^{A}, p_{1}^{B}, p_{2}^{B}\right)$. Each agent $l \in[0,1]$ from each side $i=1,2$ then chooses platform $B$ when

$$
v_{i l}+\gamma_{i} \mathbb{E}\left[q_{j}^{B}-q_{j}^{A} \mid v_{i l}\right]>p_{i}^{B}-p_{i}^{A},
$$

and platform $A$ when the above inequality is reversed. Arguments similar to those in the monopolist case then imply that, when network effects are not too large (formally when Conditions (M) and (Q) below hold), for any $p$, there is a unique continuation equilibrium and it is in threshold strategies. Each agent $l \in[0,1]$ from each side $i=1,2$ joins platform $B$ if $v_{i l}>\hat{v}_{i}$ and platform $A$ if $v_{i l}<\hat{v}_{i}$, with the thresholds $\left(\hat{v}_{1}, \hat{v}_{2}\right)$ solving the following indifference conditions

$$
\hat{v}_{i}+\gamma_{i}-2 \gamma_{i} M_{j i}\left(\hat{v}_{j} \mid \hat{v}_{i}\right)=p_{i}^{B}-p_{i}^{A} \quad i, j=1,2, j \neq i .
$$

Notice that $1-2 M_{j i}\left(\hat{v}_{j} \mid \hat{v}_{i}\right)=\mathbb{E}\left[q_{j}^{B}-q_{j}^{A} \mid \hat{v}_{i}\right]$ is the differential in the side- $j$ participation to the two platforms, as expected by the side- $i$ marginal agent with value $\hat{v}_{i}$. As we show in the Appendix (Lemma 2), the following conditions guarantee existence and uniqueness of a solution to the system of equations in (14):

Condition (M): For any $i, j=1,2, j \neq i$, any $v_{1}, v_{2} \in \mathbb{R}$,

$$
1-2 \gamma_{i} \frac{\partial M_{j i}\left(v_{j} \mid v_{i}\right)}{\partial v_{i}}>0 .
$$

Condition (Q). For any $i, j=1,2, j \neq i$, any $v_{1}, v_{2} \in \mathbb{R}$,

$$
\gamma_{1} \gamma_{2}<\frac{\left[\frac{1}{2}-\gamma_{1} \frac{\partial M_{21}\left(v_{2} \mid v_{1}\right)}{\partial v_{1}}\right]\left[\frac{1}{2}-\gamma_{2} \frac{\partial M_{12}\left(v_{1} \mid v_{2}\right)}{\partial v_{2}}\right]}{\frac{\partial M_{12}\left(v_{1} \mid v_{2}\right)}{\partial v_{1}} \frac{\partial M_{21}\left(v_{2} \mid v_{1}\right)}{\partial v_{2}}} .
$$

Condition (M) is always satisfied when preferences are aligned between the two sides. When preferences are misaligned, the condition imposes that network effects be small. This condition guarantees that a best response to agents from the opposite side following a threshold strategy is a threshold strategy. ${ }^{21}$

Condition (Q), in turn, guarantees convergence of monotone best responses to a unique fixed point by bounding the slope of best responses. Jointly, the above two conditions guarantee existence and uniqueness of a monotone continuation equilibrium. Standard arguments then imply that such equilibrium is the unique equilibrium of the continuation game.

Hereafter, we maintain that Conditions (M) and (Q) hold. Under these conditions, for any vector of prices $p$, the residual demand expected by each platform on each side $i=1,2$ is then given by $Q_{i}^{k}\left(\hat{v}_{i}\right)$, where $\left(\hat{v}_{1}, \hat{v}_{2}\right)$ is the unique solution to the equations in (14). Arguments similar to the ones discussed above for the monopolist case then lead to the following result:

\footnotetext{
${ }^{21}$ This is intuitive. If preferences are misaligned and network effects are strong, then an increase in the appreciation of platform B's product by agent $l$ from side $i$ could make the agent less willing to join the platform as a result of the fact that the agent may expect fewer agents from the opposite side to be appreciative of the platform's product and hence to join.
} 
Proposition 4. Suppose both platforms are active in the market and compete under dispersed information. The equilibrium prices, along with the participation thresholds they induce, must satisfy the following optimality conditions

$$
p_{i}^{k}=\frac{Q_{i}^{k}\left(\hat{v}_{i}\right)}{\left|d Q_{i}^{k}\left(\hat{v}_{i}\right) / d v_{i}\right|}-2 \gamma_{j} \frac{\partial M_{i j}\left(\hat{v}_{i} \mid \hat{v}_{j}\right)}{\partial v_{i}} \frac{Q_{j}^{k}\left(\hat{v}_{j}\right)}{\left|d Q_{i}^{k}\left(\hat{v}_{i}\right) / d v_{i}\right|}-2 \gamma_{i} \frac{\partial M_{j i}\left(\hat{v}_{j} \mid \hat{v}_{i}\right)}{\partial v_{i}} \frac{Q_{i}^{k}\left(\hat{v}_{i}\right)}{\left|d Q_{i}^{k}\left(\hat{v}_{i}\right) / d v_{i}\right|}
$$

along with $p_{i}^{B}-p_{i}^{A}=\hat{v}_{i}+\gamma_{i}-2 \gamma_{i} M_{j i}\left(\hat{v}_{j} \mid \hat{v}_{i}\right), i, j=1,2, j \neq i, k=A, B$.

The interpretation of the different terms in (15) is the same as in the monopoly case. The reason why all the terms involving the network effects are scaled by a factor of two is that, under singlehoming and full market coverage, the benefit of attracting more agents on board on each side $i$ combines the direct benefit of providing the side- $j$ agents with a larger user base with the indirect effect of reducing the attractiveness of the other platform.

The formulas in Proposition 4 are fairly general, in the sense that they apply to markets in which one platform is perceived as superior to the other, as well as to markets in which the information the two platforms possess need not be the same, nor need it originate from a common prior. These formulas, however, simplify significantly, permitting a close-form representation, in markets in which the two platforms perceive competition to be symmetric, in the following sense:

Definition 2. Competition is symmetric if (a) platforms set equal prices on each side (i.e., $p_{i}^{A}=p_{i}^{B}$, $i=1,2$ ) and expect to share the market evenly (i.e., $Q_{i}^{A}=Q_{i}^{B}=1 / 2, i=1,2$ ), and (b) when platforms set equal prices, all agents select the platform for which their stand-alone valuation is the highest.

Because the platforms' beliefs need not originate from a common prior, the definition does not impose any restriction on the unobserved distribution of stand-alone valuations. The following is then a direct implication of the previous proposition ${ }^{22}$ :

Corollary 1. Suppose competition is symmetric and information is dispersed. Beliefs must satisfy the following properties: (a) $Q_{i}^{k}(0)=1 / 2, k=A, B$, (b) there exists $\psi_{i}(0)>0, i=1,2$, such that $\left|d Q_{i}^{k}(0) / d v_{i}\right|=\psi_{i}(0), k=A, B$, and $(c) M_{i j}(0 \mid 0)=1 / 2, i, j=1,2, j \neq i$. The equilibrium prices are given by

$$
p_{i}^{k}=\frac{1}{2 \psi_{i}(0)}-\gamma_{j}\left[\frac{\frac{\partial M_{i j}(0 \mid 0)}{\partial v_{i}}}{\psi_{i}(0)}\right]-\gamma_{i}\left[\frac{\frac{\partial M_{j i}(0 \mid 0)}{\partial v_{i}}}{\psi_{i}(0)}\right], \quad k=A, B, i=1,2 .
$$

As in the complete-information case, equilibrium prices increase with the inverse-semi-elasticity of the component of the demand that comes from the stand-alone valuations (the first term in the price equation), and decrease with the intensity of the network effect from the opposite side. However,

\footnotetext{
${ }^{22}$ It should be obvious that the conclusions in the corollary below cease to hold if beliefs about the distribution of stand-alone valuations depend on the intensity of the network effects. When this is the case, there need not be any specific relation between the intensity of the network effects and the equilibrium prices.
} 
contrary to complete information, equilibrium prices under dispersed information increase with the intensity of the own-side network effects when preferences are aligned between the two sides, and decrease when they are misaligned. ${ }^{23}$

Hence, when competition is symmetric, each platform expects the measure of agents preferring platform $A$ 's product to platform $B$ 's product to be the same as the measure of agents preferring $B$ 's to $A$ 's (property (a) in the corollary). Furthermore, the two platforms must hold identical beliefs concerning the density of agents who are indifferent between the two platforms' products (property (b)). Finally, each agent who is just indifferent between the two platforms' products must believe that the measure of agents from the opposite side preferring platform $A$ 's product to platform $B$ 's product is the same as the measure of agents preferring platform $B$ 's product to platform $A$ 's (property (c)). ${ }^{24}$

The above results pertain to a market in which agents single-home (that is, they join only one platform) and cannot opt out of the market. In the Supplementary Material, we show that, under reasonable assumptions, the equilibrium properties identified above extend to environments in which agents can multi-home and/or opt out of the market, provided that platforms cannot set negative prices. $^{25}$

\section{Information Management}

The analysis in the previous section uncovers how equilibrium prices depend on the uncertainty that market participants face about the platforms' ability to attract participation on each side of the market. Equipped with the above results, we now investigate the platforms' incentives to invest in platform design and in various information management policies aimed at promoting their ability to attract agents from the different sides of the market. Most of such policies also affect the agents' own appreciation for the platforms' products, i.e., the level and slope of the component of the demands that comes from the stand-alone valuations. The latter effects, however, are not specific to platform markets and have received considerable attention in the literature (see, among others, Anderson and Renault (2006, 2009), and Johnson and Myatt (2006)). Hereafter, we focus on the aspects of such

${ }^{23}$ Observe that, under complete information, when competition is symmetric, the duopoly prices are given by

$$
p_{i}^{k}=\frac{1}{2 \lambda_{i}^{\theta}(0)}-\gamma_{j}, \quad k=A, B, i=1,2,
$$

where $\lambda_{i}^{\theta}(0)$ is the density of the true distribution of the side- $i$ differentials in stand-alone valuations.

${ }^{24}$ Under a common prior, these conditions are satisfied, for example, when each state is parametrized by a vector $\theta=\left(\theta_{1}, \theta_{2}\right)$ such that, given $\theta_{i}, v_{i l}=\theta_{i}+\varepsilon_{i l}$ with $\varepsilon_{i l}$ drawn independently across agents from a distribution $G_{i}$ that is symmetric around zero (i.e., such that $G_{i}(a)=1-G_{i}(-a)$ all $a \in \mathbb{R}$ ), with $\theta=\left(\theta_{1}, \theta_{2}\right)$ drawn from a distribution $F_{\theta}$ with marginals that are also symmetric around zero, as in the Gaussian model in the next section.

${ }^{25}$ See Caillaud and Jullien (2003), Armstrong and Wright (2006), Bergemann and Bonatti (2011), Athey, Calvano and Gans (2012), and Ambrus, Calvano and Reisinger (2013) for models that allow for multihoming under complete information, and Amelio and Jullien (2010) for the effects of negative prices. 
policies that are specific to platform markets. As discussed in Reillier and Reillier (2017), platforms need to innovate on their typical branding strategies to promote their ability to attract users from other sides of the market. We examine the key trade-offs that platforms face in shaping the agents' beliefs about the participation of other sides and show how the latter affect the structure of the demands and the intensity of competition among platforms.

Consistently with what we anticipated in the Introduction, we distinguish between pre-launch policies, i.e., initiatives such as marketing campaigns, the promotion of online communities, and the sponsorship of forums, blogs, and specialized conferences that platforms use at the early stages to promote their ability to attract participation from other sides of the market, and post-launch disclosures whereby platforms influence the decisions of late adopters through the dissemination of information about earlier participation decisions.

\subsection{Gaussian model}

To investigate the effects of such policies on equilibrium profits, consumer surplus, and welfare in a tractable way, we consider a market with Gaussian information. We also enrich the framework by allowing agents to possess information that is only imperfectly correlated with their own stand-alone valuations. This enrichment permits us to isolate the novel effects of information on the agents' ability to predict the participation of the other side of the market from the more familiar effects of information on the distribution of the agents' perceived valuations.

Accordingly, we distinguish between an agent's true differential in stand-alone valuations, $V_{i l}$, and an agent's estimated differential in stand-alone valuations, $v_{i l}$. We assume the "aggregate state of the world" is parametrized by a bi-dimensional vector $\theta \in \mathbb{R}^{2}$ drawn from a bi-variate Normal distribution with zero mean and given variance-covariance matrix. Each agent does not observe his true preference $V_{i l}$ but receives a signal $x_{i l}$ that is correlated with $V_{i l}$. The state of the world determines the joint distribution of true preferences and signals in the population. Precisely, we assume that, conditionally on $\theta$, each pair $\left(V_{i l}, x_{i l}\right)$ is drawn independently across agents from a bi-variate Normal distribution with fixed variance-covariance matrix and a mean given by a linear function of $\theta$. We let $v_{i l} \equiv \mathbb{E}\left[V_{i l} \mid x_{i l}\right]$ stand for an agent's estimated stand-alone differential and, without loss of generality, we then normalize the signal $x_{i l}$ so that $v_{i l}=x_{i l}$.

The above structure is common knowledge among the platforms and the agents. This implies that ex-ante (and hence, from the platforms' perspective), the joint distribution of estimated stand-alone differentials in the population is Gaussian with mean $(0,0)$ and variance-covariance matrix

$$
\Sigma_{v}=\left[\begin{array}{cc}
\left(\beta_{1}^{v}\right)^{-1} & \frac{\rho_{v}}{\sqrt{\beta_{1}^{v} \beta_{2}^{v}}} \\
\frac{\rho_{v}}{\sqrt{\beta_{1}^{v} \beta_{2}^{v}}} & \left(\beta_{2}^{v}\right)^{-1}
\end{array}\right],
$$

where $\rho_{v} \in[0,1]$ is the coefficient of linear correlation between any pair of estimated stand-alone differentials of any two agents from opposite sides and where $\beta_{i}^{v}$ is the precision of the marginal of 
the above distribution with respect to the $i^{\text {th }}$-dimension, $i=1,2 .{ }^{26}$ The marginals of this distribution with respect to each dimension $v_{i}$ thus correspond to the functions $Q_{i}^{k}$ in the general model. In particular, letting $\Phi$ denote the c.d.f. of the Standard Normal distribution (with density $\phi$ ), we have that

$$
Q_{i}^{A}\left(v_{i}\right)=\Phi\left(\sqrt{\beta_{i}^{v}} v_{i}\right)=1-Q_{i}^{B}\left(v_{i}\right) .
$$

Finally note that each agent from each side $i=1,2$ with differential $v_{i}$ believes the distribution of stand-alone differentials on the opposite side to be Gaussian with mean $\mathbb{E}\left[v_{j} \mid v_{i}\right]=\rho_{v} \sqrt{\frac{\beta_{i}^{v}}{\beta_{j}^{v}}} v_{i}$ and variance $\left(1-\rho_{v}^{2}\right) / \beta_{j}^{v} \cdot{ }^{27}$ It follows that

$$
M_{j i}\left(v_{j} \mid v_{i}\right)=\Phi\left(\sqrt{1+\Omega^{2}} \sqrt{\beta_{j}^{v}} v_{j}-\Omega \sqrt{\beta_{i}^{v}} v_{i}\right)
$$

where

$$
\Omega \equiv \frac{\rho_{v}}{\sqrt{1-\rho_{v}^{2}}}
$$

Note that $|\Omega|$ measures the sensitivity of each agent's posterior beliefs about the distribution of standalone differentials on the other side of the market to his own stand-alone differential. Equivalently, $|\Omega|$ measures the ability of each side to use individual appreciations for the platforms' products to forecast participation decisions on the opposite side of the market. The sign of $\Omega$, instead, captures the alignment in preferences across the two sides. Hereafter, we refer to the term $\Omega$ as to the coefficient of mutual forecastability and to $|\Omega|$ as the ability of each side to predict participation decisions on the opposite side of the market. ${ }^{28}$

The following proposition translates the result about pricing derived in Proposition 4 to the Gaussian environment under consideration.

Proposition 5. Suppose information is Gaussian, as described above. The duopoly equilibrium prices are then given by

$$
p_{i}^{*}=\frac{1}{2 \sqrt{\beta_{i}^{v}} \phi(0)}-\gamma_{j} \sqrt{1+\Omega^{2}}+\gamma_{i} \Omega, \quad i, j=1,2, j \neq i,
$$

where $\left(2 \sqrt{\beta_{i}^{v}} \phi(0)\right)^{-1}$ is the inverse semi-elasticity of the component of the demand curves that comes from the stand-alone valuations, and where $\Omega$ is the coefficient of mutual forecastability.

In the Gaussian case, the equilibrium price on each side thus depends on the details of the payoff and information structure only through (a) the coefficient of ex-ante dispersion of the stand-alone

\footnotetext{
${ }^{26}$ See the Supplementary Material for a flexible family of Gaussian economies satisfying all the assumptions above.

${ }^{27}$ To compute these formulas, use standard properties of the Gaussian distribution to obtain that

$$
\mathbb{E}\left[v_{j l^{\prime}} \mid v_{i l}\right]=\frac{\operatorname{cov}\left[v_{j l^{\prime}}, v_{i l}\right]}{\operatorname{var}\left[v_{i l}\right]} v_{i l}=\rho_{v} \sqrt{\frac{\beta_{i}^{v}}{\beta_{j}^{v}}} v_{i l} \text { and } \operatorname{var}\left[v_{j l^{\prime}} \mid v_{i l}\right]=\operatorname{var}\left[v_{j l^{\prime}}\right]\left(1-\rho_{v}^{2}\right)=\frac{1-\rho_{v}^{2}}{\beta_{j}^{v}}
$$

${ }^{28}$ Note that $1+\Omega^{2}=\frac{\operatorname{var}\left[v_{j}\right]}{\operatorname{var}\left[v_{j}-\mathbb{E}\left[v_{j} \mid v_{i}\right]\right]}$. Hence, for given $\operatorname{var}\left[v_{j}\right]$, a larger $|\Omega|$ implies a smaller variance of the forecast error.
} 
valuations (the term $1 / \sqrt{\beta_{i}^{v}}=\sqrt{\operatorname{var}\left[v_{i}\right]}$ in the price equations) and (b) the coefficient of mutual forecastability, $\Omega$. The sign of $\Omega$, which is the same as the sign of $\rho_{v}=\operatorname{corr}\left(v_{i}, v_{j}\right)$, reflects whether preferences are aligned or misaligned between the two sides. The sign of $\Omega$ is thus what determines whether the equilibrium price $p_{i}^{*}$ on each side increases or decreases with the intensity $\gamma_{i}$ of that side's own network effects (recall the discussion in the general model). In contrast, when it comes to the impact on equilibrium prices of the intensity of the network effects on the opposite side, $\gamma_{j}$, what matters is only $|\Omega|$. This is because the ability of the side- $j$ agents to forecast variations in the side- $i$ participation depends only on the intensity of the correlation in preferences across sides and not on its sign.

Note that a higher $\Omega$ contributes to a higher sum of the equilibrium prices. It also contributes to higher price skewness (i.e., to a higher $\left.\left|p_{1}^{*}-p_{2}^{*}\right|\right)$ if, and only if, the side with the highest price is the one on which the network effects are the largest (that is, if, and only if, $\left.\left(p_{1}^{*}-p_{2}^{*}\right)\left(\gamma_{1}-\gamma_{2}\right)>0\right)$.

\subsection{Pre-launch information management}

"Platforms should .... champion a set of clear and powerful values and a design identity that resonate with both user and producer communities." (Reillier and Reillier, 2017).

The development and launch of a new platform involves two stages. In the first stage, the developer must define the service and design the platform. In the second stage, the developer must convince enough potential users on each side to join. This second stage is often referred to as ignition (see, e.g., Evans and Schmalensee, 2015, and Reillier and Reillier, 2017, for a description of the various stages in the development of a platform). These stages involve significant uncertainty about the platform's eventual success in attracting participation on each side, as exemplified by the failure of Microsoft or RIM to promote new mobile operating systems. As a result, platforms invest significant resources in design and communication strategies aimed at aligning preferences across sides and persuading agents on each side about the appeal of the platform on the opposite side. ${ }^{29}$

In this section, we discuss a platform's incentives to affect the agents' information and the implications for welfare and consumer surplus.

From the price equations (19), we have that, in equilibrium, profits are given by

$$
\Pi^{*}=\frac{1}{2}\left(p_{1}^{*}+p_{2}^{*}\right)=\frac{1}{2}\left\{\frac{1}{2 \sqrt{\beta_{1}^{v}} \phi(0)}+\frac{1}{2 \sqrt{\beta_{2}^{v}} \phi(0)}+\left(\gamma_{1}+\gamma_{2}\right)\left(\Omega-\sqrt{1+\Omega^{2}}\right)\right\} .
$$

As for total welfare, we show in the Appendix (see the proof of Proposition 6 below) that the latter is given by

$$
W=K+\sum_{i=1,2} \mathbb{E}\left[V_{i l} \mathbb{I}\left(v_{i l}>0\right)\right]+\left(\gamma_{1}+\gamma_{2}\right) \operatorname{Pr}\left(v_{1} v_{2} \geq 0\right)
$$

\footnotetext{
${ }^{29}$ See also Lee and O'Connor (2003) for a discussion of the motives for pre-launch announcements in markets with network effects. One motive is consumers' "expectation management", another motive is the encouragement of investment in complementary products (which can be interpreted as participation on one side). A third motive is preemption.
} 
where $K$ is a constant. The term $\mathbb{E}\left[V_{i l} \mathbb{I}\left(v_{i l}>0\right)\right]$ in $W$ measures the side- $i$ 's ability to identify the platform for which the stand-alone valuation is the highest. The term $\operatorname{Pr}\left(v_{1} v_{2} \geq 0\right)$ in turn captures the agents' ability to coordinate across sides by ending up on the same platform.

Finally, consumer surplus is given by $C S=W-2 \Pi^{*}$.

The policies that most platforms use to influence the agents' participation decisions affect both the agents' ability to estimate their own stand-alone valuations as well as their ability to predict the participation decisions on the other side of the market. Enhancing the agents' ability to estimate their own valuations soften competition by increasing the heterogeneity in the agents' perceived valuations. This effect is captured by an increase in the variance $1 / \beta_{i}^{v}$ of the estimated stand-alone differentials, which unambiguously contributes to higher profits. Such effect is thus similar to the effect of an increase in the degree of differentiation between firms and is not specific to platform markets (see, for example, Johnson and Myatt (2006), and Anderson and Renault (2009)). Interestingly, when agents are imperfectly informed, such policies also improve welfare by helping the agents identify the platform for which their stand-alone valuation is the highest. This effect is captured by the first term in (21).

In the analysis below, we focus on the effects of the aforementioned policies that are specific to platform markets by analyzing how such policies affect the contribution of network effects to profits, welfare, and consumer surplus, as captured by the last terms in (20) and (21).

Proposition 6. Holding $\beta_{i}^{v}$ constant, $i=1,2$, platforms' policies that increase the alignment in preferences across sides (formally captured by an increase in $\rho_{v}$ ) increase both profits and total welfare, but decrease consumer surplus.

When preferences are aligned, an increase in $\rho_{v}$ implies a higher sensitivity of the marginal agents' expectation of the other side's participation to their own valuation (see Condition (17)). As explained in the previous section, this effect contributes to a lower elasticity of the demands and hence to higher equilibrium prices. At the same time, a higher $\rho_{v}$ also implies a higher sensitivity of the marginal agents' expectation of the other side's participation to variations in prices on the opposite side, which contributes negatively to the cross-price elasticities of the demands (see again Condition (17)). While the net effect of a higher $\rho_{v}$ on the equilibrium prices on each side depends on the relative importance that each side attaches to interacting with the opposite side, the net effect of a higher $\rho_{v}$ on the sum of the equilibrium prices and hence on total profits is unambiguously positive.

When, instead, preferences are mis-aligned, an increase in $\rho_{v}$ implies a lower sensitivity of the marginal agents' expectation of the other side's participation to their own valuations. This is because the marginal agents are more optimistic about the other side's participation than the infra-marginal agents. Thus, the larger $\left|\rho_{v}\right|$, the smaller the reduction in prices necessary to get new agents on board, and hence the larger the elasticity of the residual demands. An increase in alignment, by reducing $\left|\rho_{v}\right|$, thus contributes to a reduction in both the elasticity of the demands to their own prices and in the cross-price elasticities. As both elasticities contribute to lower prices, a higher 
alignment contributes to higher prices on both sides and hence to higher profits.

Next, consider the effect of alignment on total welfare. A higher alignment contributes to a higher probability that any two agents from opposite sides end up on the same platform (formally captured by the second term in (21)). When the sum of the network effect is positive, as assumed here, a higher alignment thus contributes unambiguously to higher welfare.

Lastly, consider the effect of alignment on consumer surplus. On the one hand, agents benefit from the enhanced ability to coordinate with agents from the opposite side. On the other hand, prices are necessarily higher at least on one side. Whether agents suffer or benefit from a higher alignment in preferences then depends on which side they belong to and on which of the above two effects prevail. Interestingly, in the Gaussian model, total consumer surplus always decreases with $\rho_{v}$.

The above conclusions extend to markets in which network effects are positive on one side but negative on the other, provided that $\gamma_{1}+\gamma_{2}>0$. When, instead, $\gamma_{1}+\gamma_{2}<0$, the above conclusions are reversed: more alignment in stand-alone valuations across the two sides contributes to lower profits and lower total welfare, but higher consumer surplus.

What is interesting about the result in Proposition 6 is that it identifies fairly general channels through which information policies aimed at aligning preferences across sides affect profits, welfare, and consumer surplus, without specifying the granular mechanics by which such policies operate. It should also be clear that, while we focus on the effects of such policies on the agents' ability to predict participation decisions on the opposite side of the market, in many cases of interest, such policies also affect the agents' ability to predict their own valuations.

There are many ways platforms may affect the alignment in preferences across sides. For our purpose, hereafter we distinguish between policies directly aimed at changing the agents' information from policies that affect directly the distribution of preferences such as platform design.

\subsubsection{Platform design}

"With platforms, a critical strategic aim is strong up-front design that will attract the desired participants, enable the right interactions (so-called core interactions), and encourage ever-more-powerful network effects." (Van Alstyne, Parker, and Choudary, Harvard Business Review (2016)).

We start by considering policies that affect the distribution from which the true stand-alone valuations are drawn. As anticipated above, such policies should be interpreted as the result of platform design. The latter has many facets. Here we focus on design decisions that affect the alignment of preferences across the two sides.

An example of such policies is the design of a collaborative architecture (see, e.g., Pisani and Verganti (2008)), that is, the process by which a users' community is allowed to contribute to the 
design of the platform through interactive communication. In the context of our illustrative example of mobile software platforms, alignment by design includes the choice by a software platform to favor aspects of its code that are considered both user- and developer-friendly, or the choice by a smartphone, or a video-game console, provider to favor dimensions such as image resolution, or low latency, that improve consumer experience but also permit developers to provide more sophisticated and flexible applications. For instance Samsung succeeded to overcome Apple's leadership by betting on larger screens, a feature that favors apps' experience and thus appeals to both end-users and developers, but which could have failed had users favored lighter handsets.

Clearly, more alignment in the true preferences need not imply more alignment in the estimated valuations, which is what platforms care about. For instance, if the side- 1 agents possess no information about the side-2 agents' preferences (which is the case when the variation in the side- 1 agents' preferences originates entirely in idiosyncratic preference shocks), then $\rho_{v}=0$. In this case, increasing the alignment in the true preferences has no effect on the correlation between the estimated differentials, and hence no effect on profit and welfare. However, in general, when each side possesses some information about the distribution of preferences on the opposite side, more alignment in the true preferences results in more alignment in the perceived preferences, and hence in higher profits and total welfare.

It is useful to compare the above results to their counterparts in standard one-sided markets. Johnson and Myatt (2006) show that, in a standard one-sided market without network effects, a monopolist maximizes profits by choosing a product design that either maximizes or minimizes the dispersion of valuations in the market. In a duopoly without network effects, equilibrium profits are always maximal under maximal dispersion, for dispersion softens competition. Our analysis reveals two departures from the optimality of maximal dispersion. First, the need to court both sides calls for a design that favors dimensions that maximize the sum of the dispersion of standalone valuations. Such design is typically different from the one that maximizes dispersion on each side. Second, platforms benefit from aligning preferences across the two sides. Once again, this is typically achieved by investing in multiple dimensions as opposed to focusing on the single dimension for which the dispersion of stand-alone differentials is maximal on each side.

The above results also bring a new perspective on the role of algorithmic price formation protocols in trading platforms, such as those used by Uber. From a welfare perspective, allowing platforms to interfere in the price formation mechanism has the benefits of permitting the various sides to adjust to variations in supply and demand conditions. From the platform's perspective, welfare-enhancing protocols bring higher profits by permitting the platform to charge higher fees. The price formation mechanism has also the additional benefit of correlating participation decisions across sides. Indeed, consider a trading platform where the two sides are buyers and sellers. Suppose that exogenous shocks to supply and demand are uncorrelated. If the platform imposes a fixed trading price, there is no correlation between the two sides' participation decisions. If, instead, the platform's transaction prices adjust to supply and demand conditions, then participation levels are correlated across the 
two sides, which brings the benefits discussed above. ${ }^{30}$

\subsubsection{Disclosures}

Think of a smart-phone company or a video-game console producer entering the market with a new operating system or a new console. The firm must decide how much information to disclose to the public about the various features of its product. These disclosures affect both the developers' and the end-users' ability to estimate their own stand-alone valuations (both in absolute value and relative to the products offered by rival firms). They also serve the purpose of persuading users and developers of the platform's ability to attract agents from the other side. As pointed by Lee and O'Connor (2003), "when network effects exist, the strategic reason for pre-announcing is to gain a faster takeoff by managing consumers' expectations". For example, prior to releasing Wii, Nintendo engaged in various marketing campaigns aimed at promoting the new console to both potential gamers and developers (see Sterlicchi (2013)). Representatives were hired to provide interactive demos at home-shows, fitness expos, and major malls. As reported in Chellappa and Mukherjee (2017), such pre-launch initiatives are believed to have played a major role in Wii's success. On the other hand, the specialized press has often criticized Microsoft for not providing the market with enough details regarding its Xbox One, thus failing to persuade gamers and developers of the network's potentiality of its console (see, Gallaway (2013), and Chellappa and Mukherjee, (2017)).

Another example of such pre-launch campaigns is RIM's decision the year preceding the launch of its Blackberry 10 to give away thousand of prototypes phones to developers. ${ }^{31}$ A key motivation was to overcome its reputation of being unable to deliver promised quality to consumers. RIM followed a similar strategy for its PlayBook tablet.

We think of pre-launch disclosures as part of a broader class of information management policies aimed at influencing the agents' participation decisions (referred to as psychological positioning by Arthur (1996)). Such policies also include the sponsorship of forums, blogs, and conferences that platforms use to cultivate and manage expectations. For example, Google uses its portal Google Android Authority to promote communication between end-users and app developers. Likewise, Apple uses its annual Apple World Wide Developer Conference to help iOS developers predict endusers' interests (see, e.g., Förderer (2017)).

Following the terminology of Johnson and Myatt (2006), we distinguish between "hypes" and "real information." The former refer to persuasive campaigns that change the level of demand expected by the platforms. The latter, instead, refer to campaigns that do not change the level of demand, on average, but affect the dispersion of perceived valuations and of beliefs about the other side's participation. We focus on these latter campaigns. Such campaigns may operate along various dimensions and may involve advertising, blogs, forums, and the release of prototypes and freeware.

\footnotetext{
${ }^{30}$ See also Castillo, Knoepfle, and Weyl (2018) for an analysis of the benefits of algorithmic pricing in the context of share riding.

${ }^{31}$ See Austen (2012), "BlackBerry 10 Prototype Is Given To Developers", The New York Times.
} 
Consistently with the analysis above, we abstain from the granular mechanics of such campaigns and isolate the novel effects specific to platform markets by focusing on how variations in the agents' ability to predict the participation of the other side affects profits, welfare and consumer surplus.

Proposition 7. Suppose preferences are aligned across sides. Holding $\beta_{i}^{v}$ fixed, $i=1,2$, information policies that increase the agents' ability to predict participation decisions on the opposite side of the market (formally captured by an increase in $|\Omega|$ ) increase profits and total welfare but reduce consumer surplus. When, instead, preferences are misaligned, such policies decrease profits and total welfare but increase consumer surplus.

Consider campaigns on side $i$ that, by providing information about features of the platform relevant for the other side, reduce the side- $i$ agents' idiosyncratic interpretation of what is likely to be appreciated by the agents on side $j$. As mentioned above, an example of such campaigns is Apple's use of its annual World Wide Developers' conference as a means to provide developers with information about new market niches for its mobile operating system (see, e.g., Förderer (2017)). When preferences are aligned, that is, when $\rho_{v}>0$, such campaigns, by increasing the correlation in the estimated stand-alone valuations across the two sides, contribute to higher profits and total welfare. The opposite is true when preferences are mis-aligned. In this case, an increase in the agents' ability to interpret what is likely to be hip on the opposite side implies a reduction in the preferences alignment across the two sides (formally, a reduction in $\rho_{v}$ ). In this case, information about preferences on the opposite side contributes to lower profits and total welfare.

We should point out that providing agents with more information does not necessarily mean improving their ability to predict the other side's participation. To see this, assume that the true stand-alone differentials are given by $V_{i l}=\theta_{i}+\varepsilon_{i l}$, where $\varepsilon_{i l}$ is a taste shock capturing idiosyncratic variation in the preferences of the side- $i$ agents. Suppose agents do not observe $V_{i l}$ but, instead, receive information summarized in the signal $x_{i l}=\theta_{i}+\alpha \varepsilon_{i l}+\eta_{i l}$, where $\eta_{i l}$ is Gaussian white noise. Here $\alpha$ measures the degree of congruence between the agents' true stand-alone differentials and their estimated ones, while $\eta_{i l}$ is an error term. Information that reduces the variance of $\eta_{i l}$ increases the agents' ability to predict both their true preferences, $V_{i l}$, as well as the preferences on the other side of the market (insofar $\theta_{j}$ is correlated with $\theta_{i}$ ). Instead, information that increases $\alpha$ may increase the agents' ability to understand their own preferences but at the cost of reducing their ability to predict the participation of the other side.

More generally, because both the dispersion of the estimated stand-alone differentials and the alignment in preferences across the two sides contribute to higher profits and because information that increases the former may reduce the latter, platforms may face non-trivial trade-offs when it comes to designing their communication strategies. 


\subsubsection{Word of mouth and viral communications}

Recent technological developments have provided platforms with many new opportunities for marketing and communications. In addition to traditional promotion campaigns, platforms now use online communities and social networks to promote their services through viral communication (see, e.g., Aral and Walker (2011)). A concern is that platforms loose control over the content of such viral communication (see, e.g., Godes et al (2005), and Aral, Dellarocas and Godes (2013)).

For our concerns, word of mouth is a form of information sharing that may mitigate the dispersion of beliefs. Depending on the nature of the community, the type of dispersion concerned may vary. For instance, a community restricted to one side may improve primarily that side's agents understanding of their own preferences, with little effect on these agents' ability to predict preferences on the other sides. In contrast, a community open to both sides, such as a forum designed to attract agents from both sides of the market, or a viral advertising campaign on a social network that attracts users from both sides (for instance, potential buyers and sellers of a $\mathrm{C} 2 \mathrm{C}$ website) may lead to a better understanding of features that appeal to both sides, although, in some cases, this may come at the cost of reducing the dispersion of beliefs within a given side. Whether platforms should promote one-sided or two-sided communities then crucially depends on the alignment in preferences across the two sides, as indicated by the results above.

\subsection{Post-launch disclosures}

Following the launch of a new smartphone, or a new video-game console, the evolution of sales is highly scrutinized and reported by the specialized press. Platforms themselves often report adoption rates on both sides of the market. Such post-launch disclosures, when credible, provide agents with information about the actual participation of the opposite side allowing them to adjust their behavior. They differ from the pre-launch campaign discussed above whose role is to improve the agents' ability to use their own appreciation for the platforms' products to predict participation decisions on the opposite side of the market.

In this section we investigate how post-launch disclosures affect profits and welfare. Intuitively, as end-users and developers obtain information in addition to their own valuation, we expect such post-launch disclosures to improve the coordination of the two sides and to change the demand elasticities.

To investigate the effects of such post-launch disclosures on profits in the simplest possible way, we consider a dynamic extension in which agents can change platforms after receiving information about the earlier participation decisions. ${ }^{32}$ We assume there are two periods, and allow agents to switch platforms in the second period. We assume that switching costs are negligible and disregard them. The cross-sectional distribution of stand-alone valuations is constant over the two periods.

\footnotetext{
${ }^{32}$ In this section, we focus on the effects of such policies on profits. That such policies contribute positively to welfare is immediate given that they permit agents to better coordinate across sides.
} 
Information is dispersed in the first period but agents observe the first-period aggregate participation decisions prior to making their second-period decisions. For simplicity, we assume that preferences are time-separable (i.e., the sum of the flow payoffs). Agents who do not change platform must still renew their membership in the second period by paying to the platform a participation fee in the second period.

To illustrate the key effects in the simplest possible way, we assume platforms maintain prices constant over the two periods (the alternative case in which platforms adjust prices at the same frequency at which agents revise their beliefs is examined in the Supplementary Material). To ease the exposition, we maintain the assumptions of the Gaussian model but assume that agents face no uncertainty about their own stand-alone valuations, i.e., $V_{i l} \equiv v_{i l}$ for all agents. The equilibrium characterization in this section, however, does not depend on the distributions being Gaussian. What is important is that, for any given price vector, there is a one-to-one mapping between the aggregate state of the world $\theta$ and the realized demands. This property is guaranteed when network effects are small. In particular, we assume that $\gamma_{1}$ and $\gamma_{2}$ are such that, for any state $\theta$,

$$
\gamma_{1} \gamma_{2}<\frac{1}{4 \max _{x} \lambda_{1}^{\theta}(x) \max _{x} \lambda_{2}^{\theta}(x)},
$$

where $\lambda_{i}^{\theta}$ is the density of the true distribution $\Lambda_{i}^{\theta}$ of stand-alone differentials in state $\theta$.

Because switching costs are negligible, given any collection of prices $p=\left(p_{1}^{A}, p_{2}^{A}, p_{1}^{B}, p_{2}^{B}\right)$, the period-1 demands expected by the two platforms continue to be given by $Q_{i}^{A}\left(\hat{v}_{i}\right)$ and $Q_{i}^{B}\left(\hat{v}_{i}\right)$, with the vector of thresholds $\hat{v}=\left(\hat{v}_{1}, \hat{v}_{2}\right)$ given by (14). The observation of the aggregate period-1 participation decisions then perfectly reveals to each agent the aggregate state $\theta$. The period-2 demands are then given by $q_{i}^{A}(\theta)=\Lambda_{i}^{\theta}\left(v_{i}^{\theta}\right)$ and $q_{i}^{B}(\theta)=1-\Lambda_{i}^{\theta}\left(v_{i}^{\theta}\right)$, with the thresholds $v^{\theta}=\left(v_{1}^{\theta}, v_{2}^{\theta}\right)$ solving ${ }^{33}$

$$
v_{i}^{\theta}+\gamma_{i}-2 \gamma_{i} \Lambda_{j}^{\theta}\left(v_{j}^{\theta}\right)=p_{i}^{B}-p_{i}^{A}, \quad i, j=1,2, j \neq i .
$$

Combining (14) with (23), we can express the period-2 participation thresholds as a deterministic function of the period-1 participation thresholds, with the functions $v_{i}^{\theta}(\hat{v})$ implicitly given by the (unique) solution to the system of equations given by

$$
v_{i}^{\theta}=\hat{v}_{i}-2 \gamma_{i}\left[M_{j i}\left(\hat{v}_{j} \mid \hat{v}_{i}\right)-\Lambda_{j}^{\theta}\left(v_{j}^{\theta}\right)\right], \quad i, j=1,2, j \neq i .
$$

This representation of the period-2 thresholds is convenient because it permits us to express the platforms' intertemporal expected demands

$$
\mathbf{Q}_{i}^{A}(\hat{v})=Q_{i}^{A}\left(\hat{v}_{i}\right)+\mathbb{E}_{\theta}\left[q_{i}^{A}(\theta)\right]=\mathbb{E}_{\theta}\left[\Lambda_{i}^{\theta}\left(\hat{v}_{i}\right)+\Lambda_{i}^{\theta}\left(v_{i}^{\theta}(\hat{v})\right)\right]=2-\mathbf{Q}_{i}^{B}(\hat{v})
$$

as a function of the period-1 participation thresholds. The key difference with respect to the static model is that the participation that each platform now expects from side $i$ over the two periods

\footnotetext{
${ }^{33}$ As shown in Lemma 1 in the Appendix, the system of equations given by (23) admits a unique solution, for any $\theta$, when Condition (22) holds. Note that this latter condition is stronger than Condition Q in the previous section.
} 
depends not only on the side- $i$ period- 1 marginal consumer, $\hat{v}_{i}$, but also on the side- $j$ 's period- 1 marginal consumer, $\hat{v}_{j}$.

Following steps similar to those in the baseline model (see Part 1 in the proof of Proposition 8 in the Appendix), we then have that the equilibrium prices, along with the period-1 participation thresholds they induce, must solve the following optimality conditions

$$
\begin{aligned}
p_{i}^{A} & =\frac{\mathbf{Q}_{i}^{A}(\hat{v})}{\partial \mathbf{Q}_{i}^{A}(\hat{v}) / \partial v_{i}}-2 \gamma_{j} \frac{\partial M_{i j}\left(\hat{v}_{i} \mid \hat{v}_{j}\right)}{\partial v_{i}} \frac{\mathbf{Q}_{j}^{A}(\hat{v})}{\partial \mathbf{Q}_{i}^{A}(\hat{v}) / \partial v_{i}}-2 \gamma_{i} \frac{\partial M_{j i}\left(\hat{v}_{j} \mid \hat{v}_{i}\right)}{\partial v_{i}} \frac{\mathbf{Q}_{i}^{A}(\hat{v})}{\partial \mathbf{Q}_{i}^{A}(\hat{v}) / \partial v_{i}} \\
& -p_{j}^{A} \frac{\partial \mathbf{Q}_{j}^{A}(\hat{v}) / \partial v_{i}}{\partial \mathbf{Q}_{i}^{A}(\hat{v}) / \partial v_{i}},
\end{aligned}
$$

along with (14). Similar conditions hold for platform $B$. The above pricing equations are qualitatively similar to the ones in the baseline model, except for one important difference. After seeing the participation decisions in the first period, agents learn the distribution of stand-alone differentials and use such information in the second period to revise their decisions. When this is the case, it is not possible for the platforms to adjust the prices so as to perfectly neutralize the effect of a variation in the expected participation of one side on the expected participation of the opposite side over both periods. In particular, changing the price on side $i$ and adjusting the price on side $j$ so as to maintain the side- $j$ 's period-1 participation constant does not guarantee that the side- $j$ 's period-2 participation also remains constant. This novel effect is captured by the last term in the price equation (24), which is absent in the static benchmark.

Importantly, this novel effect has implications for equilibrium profits. To see this in the simplest possible way, suppose that competition is symmetric, in the sense that the two platforms set the same prices on each side and expect, on average, (a) an equal share of the market in each period and (b) agents to favor in period one the platform for which their stand-alone valuation is the highest. In this case, the above optimality conditions imply that, in equilibrium, prices are given by

$$
\begin{aligned}
p_{i}^{* *} & =\frac{1}{\partial \mathbf{Q}_{i}^{A}(0,0) / \partial v_{i}}\left\{1-2 \gamma_{j} \frac{\partial M_{i j}(0 \mid 0)}{\partial v_{i}}-2 \gamma_{i} \frac{\partial M_{j i}(0 \mid 0)}{\partial v_{i}}\right\} \\
& -p_{j}^{* *} \frac{\partial \mathbf{Q}_{j}^{A}(0,0) / \partial v_{i}}{\partial \mathbf{Q}_{i}^{A}(0,0) / \partial v_{i}},
\end{aligned}
$$

for $i, j=1,2, j \neq i$, where

$$
\partial \mathbf{Q}_{i}^{A}(0,0) / \partial v_{i}=\mathbb{E}_{\theta}\left[\lambda_{i}^{\theta}(0)\right]+\mathbb{E}_{\theta}\left[\lambda_{i}^{\theta}\left(v_{i}^{\theta}(0,0)\right) \partial v_{i}^{\theta}(0,0) / \partial v_{i}\right]
$$

and

$$
\partial \mathbf{Q}_{j}^{A}(0,0) / \partial v_{i}=\mathbb{E}_{\theta}\left[\lambda_{j}^{\theta}\left(v_{j}^{\theta}(0,0)\right) \partial v_{j}^{\theta}(0,0) / \partial v_{i}\right]
$$

are the marginal variations in the two sides' intertemporal demands triggered by a joint variation in prices that leaves the side- $j$ 's period-1 demands unchanged.

To understand how post-launch disclosures affect profits suppose that, instead, platforms do not disclose at the end of the first period any information about the period- 1 participation decisions. 
Apart from this change, the environment is the same as in the rest of this subsection. In particular, agents must continue to choose which platform to join in each of the two periods. However, because they receive no information at the end of the first period, in equilibrium, no agent changes platform in the second period. That is, in the absence of any post-launch disclosure, $v_{i}^{\theta}(\hat{v})=\hat{v}_{i}$, for all $\theta$, $i=1,2$, in which case the intertemporal demands are given by $2 Q_{i}^{A}(0)=2 \mathbb{E}_{\theta}\left[\Lambda_{i}^{\theta}(0)\right]$. It is then immediate that, in the absence of post-launch disclosures, the equilibrium prices are same as in the static benchmark in the previous section. In particular, when all players share a common prior, as assumed in this section, the equilibrium prices without post-launch disclosure are given by

$$
p_{i}^{*}=\frac{1}{2\left[\partial Q_{i}^{A}(0) / \partial v_{i}\right]}\left\{1-2 \gamma_{j} \frac{\partial M_{i j}(0 \mid 0)}{\partial v_{i}}-2 \gamma_{i} \frac{\partial M_{j i}(0 \mid 0)}{\partial v_{i}}\right\},
$$

$i, j=1,2, j \neq i$, with $\partial Q_{i}^{A}(0) / \partial v_{i}=\mathbb{E}_{\theta}\left[\lambda_{i}^{\theta}(0)\right]$. Comparing the equilibrium prices across the two environments, we then obtain the following result:

Proposition 8. Suppose that competition is symmetric and that, in the absence of any disclosure, platforms set positive prices on both sides of the market. (a) Post-launch disclosures increase profits if, starting from the equilibrium prices without disclosure, joint variations in prices across sides that boost the period-1 demand on one side leaving the period-1 demand on the opposite side constant increase each side's demand in period two less than in period one; a sufficient condition for this to happen is that $d_{i} \equiv \mathbb{E}_{\theta}\left[\lambda_{i}^{\theta}\left(v_{i}^{\theta}(0,0)\right) \partial v_{i}^{\theta}(0,0) / \partial v_{i}\right]-\mathbb{E}_{\theta}\left[\lambda_{i}^{\theta}(0)\right] \leq 0$ and $c_{j i} \equiv \mathbb{E}_{\theta}\left[\lambda_{j}^{\theta}\left(v_{j}^{\theta}(0,0)\right) \partial v_{j}^{\theta}(0,0) / \partial v_{i}\right] \leq 0$. (b) Post-launch disclosures decrease profits if the same joint variations in prices as in part (a) above increase each side's demand in period two more than in period one.

Consider a market in which, in the absence of post-launch disclosures, platforms set positive prices on both sides (recall that this is always the case when network effects are not too large, i.e., when $\gamma_{1}$ and $\gamma_{2}$ are small). Without loss of generality, take the perspective of platform $A$ and observe that joint variations in $p_{1}^{A}$ and $p_{2}^{A}$ that boost the period- 1 demand on side $i$ while leaving the period-1 demand on side $j$ constant amount to an increase in the period-1 participation threshold $\hat{v}_{i}$ for fixed period- 1 side- $j$ threshold $\hat{v}_{j}$. In the absence of post-launch disclosures, because no new information is revealed at the end of the first period, such joint price variations trigger an equal increase in the side- $i$ 's participation to platform $A$ over the two periods equal to $\mathbb{E}_{\theta}\left[\lambda_{i}^{\theta}(0)\right]$, where the latter is simply the mass of agents who are ex-ante expected to be indifferent between the two platforms. Furthermore, by construction, such variations, on average, leave the side- $j$ participation to platform $A$ unchanged in both periods.

Now consider the same joint price variations under post-launch disclosures. Because there are no switching costs, the period-1 variation in each of the two side's participation to platform $A$ expected by the platform is the same as in the absence of post-launch disclosures. This is not the case, for period 2. Under post-launch disclosures, the platform expects a variation in the period-2 participation by side $i$ equal to $\mathbb{E}_{\theta}\left[\lambda_{i}^{\theta}\left(v_{i}^{\theta}(0,0)\right) \partial v_{i}^{\theta}(0,0) / \partial v_{i}\right]$ and a variation in the period-2 participation by side $j$ 
equal to $\mathbb{E}_{\theta}\left[\lambda_{j}^{\theta}\left(v_{j}^{\theta}(0,0)\right) \partial v_{j}^{\theta}(0,0) / \partial v_{i}\right]$. To understand, observe that $\lambda_{i}^{\theta}\left(v_{i}^{\theta}(0,0)\right)$ is the mass of agents who, in state $\theta$, are indifferent between joining one platform or the other when the two platforms set equal prices, whereas $\partial v_{i}^{\theta}(0,0) / \partial v_{i}$ is the variation in the side- $i$ period- 2 threshold triggered by a marginal variation in the side- $i$ period- 1 threshold (equivalently, by a marginal variation in the side- $i$ period-1 demand). When the above period-2 variations are smaller than their period-1 counterparts, the platform's incentives to cut its side- $i$ price are smaller under post-launch disclosures than in their absence. As a result, in equilibrium, prices, and hence profits, are higher under post-launch disclosures than in their absence.

The above conclusions are reversed when the variation in the period- 2 demands expected by the platform is larger than the variation in the period- 1 demands, on both sides of the market. In this case, post-launch disclosures contribute negatively to profits ex-ante.

To better appreciate how post-launch disclosures affect prices and profits, consider again the Gaussian model. With post-launch disclosures, the equilibrium prices cannot be computed in closed form, so we resort to numerical methods to illustrate the key findings (see also part 3 in the proof of Proposition 8 for an illustration of how the conditions in Proposition 8 specialize to the Gaussian model). Consider a market whose primitives are symmetric across sides. Specifically, assume each $\theta_{i}$ is drawn from a Gaussian distribution with mean zero and precision $\beta^{\theta}, i=1,2$, and let $\rho_{\theta}$ denote the coefficient of linear correlation between $\theta_{1}$ and $\theta_{2}$. Given $\theta=\left(\theta_{1}, \theta_{2}\right)$, the stand-alone differential for each agent $l \in[0,1]$ from each side $i=1,2$ is given by $v_{i l}=\theta_{i}+\varepsilon_{i l}$ with each $\varepsilon_{i l}$ drawn independently across agents and independently from $\theta$ from a Gaussian distribution with mean zero and precision $\beta^{\varepsilon}$. Figures 1 and 2 plot the difference

$$
\Delta \equiv p_{1}^{* *}+p_{2}^{* *}-\left(p_{1}^{*}+p_{2}^{*}\right)
$$

in the sum of the equilibrium prices, with and without post-launch disclosures, as a function of the intensity of the network effects $\gamma=\gamma_{1}=\gamma_{2}$, for different levels of $\left(\rho_{\theta}, \beta^{\varepsilon}, \beta^{\theta}\right)$. Note that, because competition is symmetric, the difference in profits coincides with the difference in prices.

As the figures illustrate, prices are higher under post-launch disclosures, and the more so the larger the network effects. When earlier participation decisions are observable, in later periods, agents use the adoption rates in earlier periods, as opposed to their own appreciation for the platforms' products, to form beliefs about the platforms' ability to attract users in the subsequent periods. This channel contributes to a reduction in the correlation between the agents' own stand-alone valuations and the expected network effects. Because such correlation contributes to a reduction in the elasticity of the demands, through this channel, post-launch disclosures contribute to a higher demand elasticity and hence to lower prices. At the same time, post-launch disclosures also permit agents from different sides to better coordinate on which platform to join. More coordination softens competition by making the two platforms more heterogenous ex-post and is thus akin to a higher degree of horizontal differentiation, but originating in network effects. Through this second channel, post-launch disclosures contribute to higher prices. In the Gaussian model, this second effect prevails, 


$$
\beta^{\theta}=1.0
$$
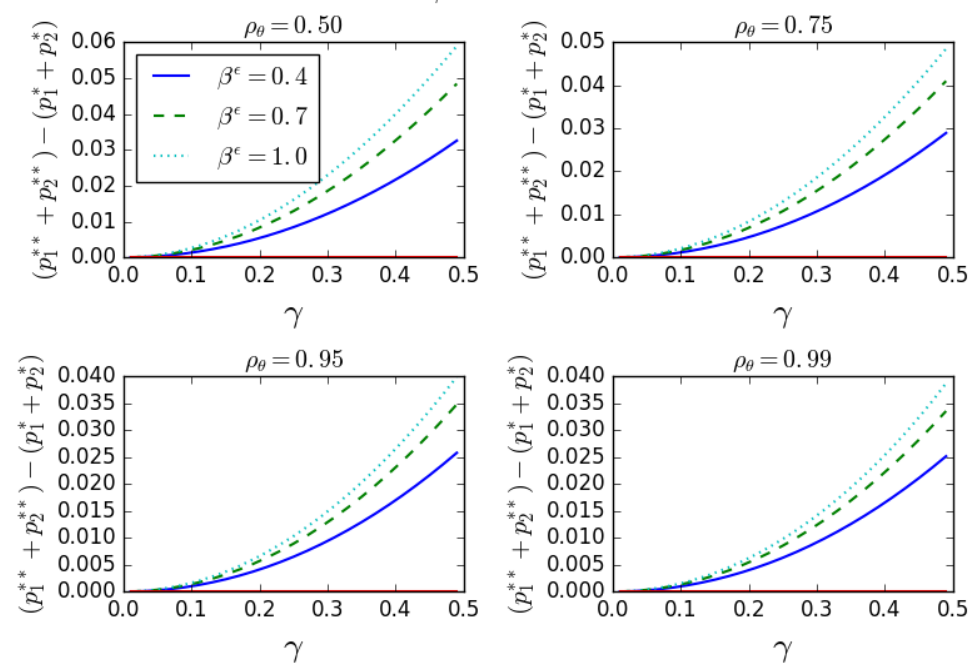

Figure 1: Price differential as a function of network effects (large uncertainty)

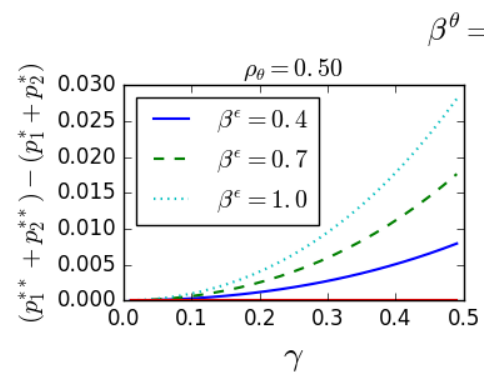

$$
\beta^{\theta}=10.0
$$
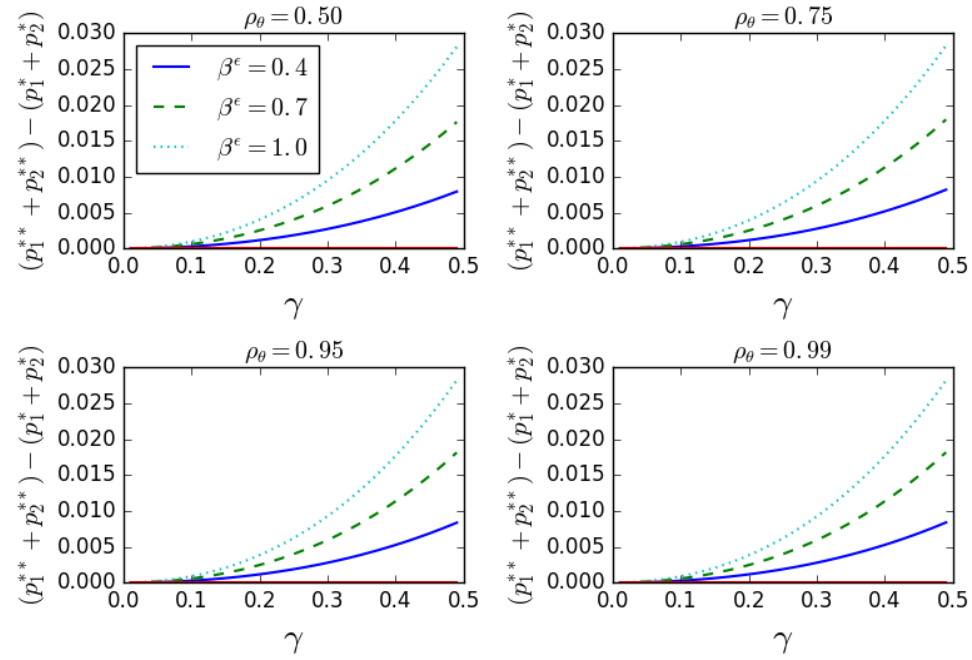

Figure 2: Price differential as a function of network effects (small uncertainty) 

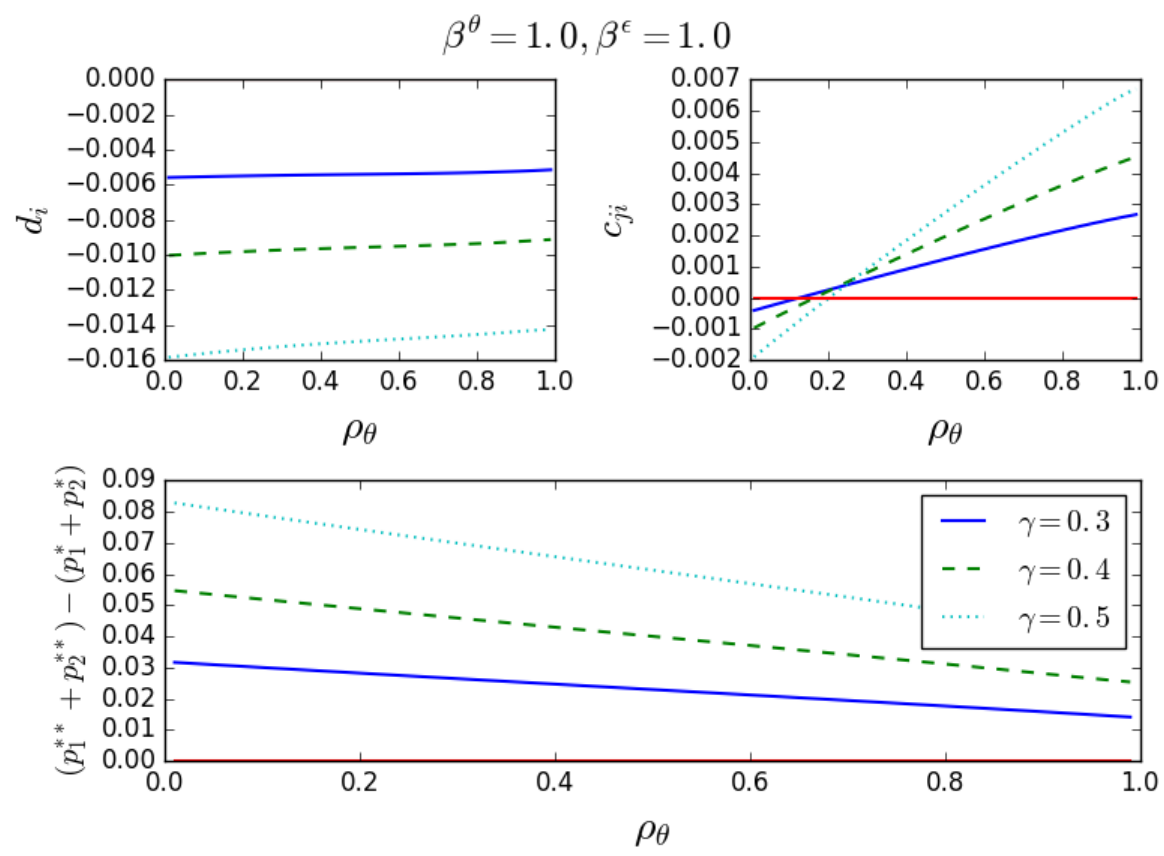

Figure 3: Price differential as a function of preference alignment (large uncertainty)

making prices higher under post-launch disclosures. Clearly, the smaller the ex-ante uncertainty (i.e., the larger $\beta^{\theta}$ is), the smaller the effect of post-launch disclosures on the expected variation in the period-2 demands and hence the smaller the effect of such disclosures on the difference $\Delta$ in the equilibrium prices across the two environments, as one can see by comparing the graphs in Figure 1 with their counterparts in Figure 2. Likewise, the larger $\beta^{\varepsilon}$ is, the smaller the idiosyncratic variation in the agents' appreciation for the two platforms and hence the stronger the benefit of letting the agents coordinate across sides by observing the earlier participation decisions.

Figures 3 and 4 illustrate a similar pattern but from a different angle. They depict the relationship between the price differential $\Delta$ and the coefficient of linear correlation $\rho_{\theta}$, for different specifications of $\gamma$ and $\beta^{\theta}$. They also decompose the effects of variations in $\rho_{\theta}$ on $\Delta$ into the two forces identified in Proposition 8, namely into $d_{i}$ and $c_{j i}$. Recall that the terms $d_{i}$ measure the difference in the period-2 marginal demands (with and without post-launch disclosures) stemming from joint price variations that leave the period- 1 side- $j$ demand constant. The terms $c_{j i}$, in contrast, measure the analog differential in period-2 demand on side $j$.

In the Gaussian model, a higher $\rho_{\theta}$ implies a higher alignment in preferences across sides, for fixed ex-ante dispersion of stand-alone differentials, $\beta_{i}^{v}$. When uncertainty is large (Figure 3), more alignment in preferences unambiguously contributes to a smaller price differential $\Delta$. While prices, and hence profits, are always higher with post-launch disclosures than in their absence, the benefits 

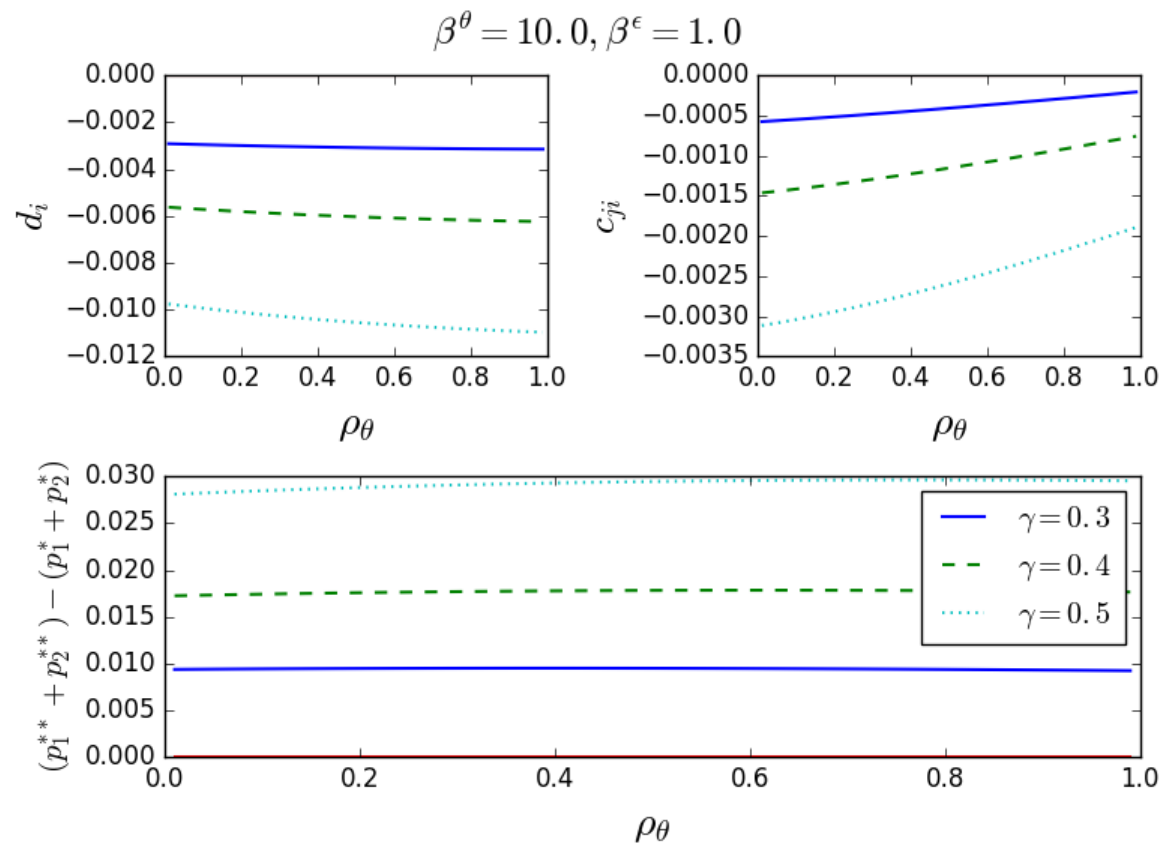

Figure 4: Price differential as a function of preference alignment (small uncertainty)

of post-launch disclosures decline with the alignment of preferences across sides. Interestingly, when ex-ante uncertainty is large and preferences are sufficiently aligned, the two forces identified in Proposition 8 contribute in the opposite direction to the price differential $\Delta$ : joint variations in prices that increase the side- $i$ period -1 demand while leaving the side- $j$ period- 1 demand constant contribute to a smaller increase in the side- $i$ period- 2 demand than in the absence of post-launch disclosures but to a larger increase in the side- $j$ period- 2 demand $\left(d_{i}<0\right.$ but $c_{j i}>0$, for large $\left.\rho_{\theta}\right)$. Furthermore, the larger $\rho_{\theta}$, the larger $d_{i}$ and $c_{j i}$, implying a stronger incentive to cut prices under post-launch disclosures. The above effects are dampened when there is little uncertainty from an ex-ante perspective, i.e., when $\beta^{\theta}$ is large (Figure 4). Furthermore, in this case, the two forces identified in Proposition 8 contribute both to higher prices under post-launch disclosures (both $d_{i}$ and $c_{j i}$ are negative in this case). However, while the effect of the first channel, $d_{i}$, declines with the alignment in preferences across sides, the effect of the second channel, $c_{j i}$, increases with $\rho_{\theta}$.

To understand why the benefit of post-launch disclosures may decrease with the alignment in preferences across sides, note that, on the one hand, more alignment contributes to a higher coefficient of mutual forecastability $\Omega$ and hence to a smaller elasticity of the demands in the absence of postlaunch disclosures. Through this channel, more alignment contributes to a smaller differential $\Delta$ and hence to a smaller benefit of post-launch disclosures. On the other hand, more alignment also contributes to higher "tipping" ex-post and hence to a higher impact of post-launch disclosures on the platforms' differentiation. Through this second channel, more alignment contributes to a higher $\Delta$. 
What Figures 3 and 4 reveal is that, in the Gaussian model, when the degree of ex-ante uncertainty is large, the first channel tends to prevail, whereas the two channels tend to cancel each other out when the ex-ante degree of uncertainty is small.

We conducted a large set of simulations and found that patters similar to the ones in the above figures emerge across a variety of parameters' specifications, including those for markets whose primitives are asymmetric across sides (the results are available upon requests). Importantly, the above results do not hinge on the agents learning perfectly the distribution of preferences by observing the earlier participation decisions. The same conclusions obtain when the earlier participation decisions are observed with noise, as is likely to be the case in most markets of interest. Finally note that the above results have implications also for mature markets where the demand on each side fluctuates over time as the result of shocks to the distribution of preferences. In such markets, platforms tend to benefit from disclosing promptly variations in the actual participation of the two sides when network effects are strong and preferences are not too aligned across sides.

\section{Conclusions}

The analysis delivers a few important lessons for platform markets. First, dispersed information introduces correlation between the agents' appreciations for a platform's product and their beliefs about the platform's ability to attract users from other sides of the market. When preferences are aligned across sides, such correlation contributes to a lower elasticity of the residual demands and to higher equilibrium prices. Second, platforms benefit from aligning preferences across sides, for example through design that favors product dimensions expected to be appreciated by both sides, or the promotion of collaborative architectures that permit agents from multiple sides to shape the platform's product. Third, once preferences are aligned, platforms benefit from engaging in various pre-launch campaigns whose role is to encourage agents to use their own appreciations to predict the participation of the other sides. Such campaigns, however, are not guaranteed to favor consumers, for, by softening competition, they permit platforms to charge higher prices. Fourth, in dynamic markets, the benefits platforms derive from disclosing information about earlier participation decisions are stronger the larger the network effects but may decline with the alignment in preferences across sides. These results can help appreciate the importance platforms assign to the promotion of showrooms, trials, forums, architectural design, and viral marketing campaigns at the early stages, when igniting the market is essential to their survival.

In future work, it would be interesting to extend the analysis to richer dynamic environments and investigate platforms' incentives to shape the dynamics of individual and social learning. It would also be interesting to accommodate for price discrimination and study how platforms grant differential access to the participating agents from other sides of the market. ${ }^{34}$ Finally, it would be

\footnotetext{
${ }^{34}$ See Damiano and Li (2007), Gomes and Pavan (2016, 2017), and Veiga, White, and Weyl (2017) for models that combine certain forms of price discrimination with heterogeneity in attractiveness. See also Ambrus and Argenziano
} 
interesting to introduce richer forms of preference heterogeneity and investigate the implications of decreasing returns to scale to network effects.

One aspect of information management that we did not consider in this paper relates to platforms' practice of artificially inflating agents' beliefs about participation levels through the creation of fake profiles (see, e.g., the discussion of Reddit, a social news aggregator, in Reillier and Reillier, 2017). We plan to examine this extension as well as the other avenues indicated above in future research.

\section{References}

[1] van Alstyne, M., G. Parker, and S. Choudary (2016): "Pipelines, Platforms, and the New Rules of Strategy," Harvard Business Review. Vol. 94, Issue 4.

[2] Ambrus, A., Calvano E. and M. Reisinger (2013): "Either or Both Competition: A Two-sided Theory of Advertising with Overlapping Viewerships," Working Paper, Duke University.

[3] Ambrus, A. and R. Argenziano (2009): "Asymmetric Networks in Two-Sided Markets," American Economic Journal: Microeconomics, 1(1) , 17-52.

[4] Amelio A. and B. Jullien (2012): "Tying and Freebies in Two-sided Markets," International Journal of Industrial Organization, 30(5), 436-446.

[5] Anderson, S. and R. Renault (2006): "Advertising Content," American Economic Review 96,(1), 93-113.

[6] Anderson, S. and R. Renault (2009): "Comparative Advertising: Disclosing Horizontal Match Information," RAND Journal of Economics, 40,(3), 558-581.

[7] Aral, S., Dellarocas, C., \& Godes, D. (2013): "Introduction to the special issue - social media and business transformation: a framework for research," Information Systems Research, 24(1), 3-13.

[8] Aral, S., \& Walker, D. (2011): "Creating social contagion through viral product design: A randomized trial of peer influence in networks," Management science, 57(9), 1623-1639.

[9] Argenziano, R. (2008): "Differentiated Networks: Equilibrium and Efficiency" Rand Journal of Economics 39, 747-769.

[10] Armstrong, M. (2006): "Competition in Two-Sided Markets," Rand Journal of Economics 37, 668-691.

(2009) for a model with heterogenous network effects in which platforms discriminate by offering multiple networks. 
[11] Armstrong, M. and J. Wright (2007): "Two-Sided Markets, Competitive Bottlenecks, and Exclusive Contracts," Economic Theory 32, 353-380.

[12] Arthur, B. W. (1996): "Increasing Returns and the New World of Business," Harvard Business Review 74 (4): 100-109.

[13] Athey, S., E. Calvano and J.S. Gans (2012): "The Impact of the Internet on Advertising Markets for News Media,"Working Paper, Harvard University.

[14] Bergemann, D. and A. Bonatti (2011): "Targeting in Advertising Markets: Implications for Offline versus Online Media," RAND Journal of Economics, 42, 417-443.

[15] Boudreau, K. and A. Hagiu, (2009): "Platform Rules: Multi-Sided Platforms as Regulators," in Platforms, Markets and Innovation, edited by A. Gawer, Edward Elgar Publishing.

[16] Cabral L. (2011): "Dynamic Price Competition with Network Effects", Review of Economic Studies 78 (1): 83-111.

[17] Caillaud, B. and B. Jullien (2001): "Competing Cybermediaries," European Economic Review (Papers \& Proceedings) 45, 797-808.

[18] Caillaud, B. and B. Jullien (2003): "Chicken \& Egg: Competition Among Intermediation Service Providers," Rand Journal of Economics, 34, 309-328.

[19] Castillo, J.C., D. Knoepfle, and G. Weyl: "Surge Pricing Solves the Wild Goose Chase" mimeo Microsoft Research.

[20] Carlsson, H., and E. Van Damme (1993): "Global Games and Equilibrium Selection," Econometrica 61, 989-1018.

[21] Chellappa, R., and R. Mukherjee (2017): "Platform Preannouncement Strategies: The Strategic Role of Information in Two-Sided Markets Competition," mimeo Emory University.

[22] Damiano, E. and H. Li (2007): "Price Discrimination and Efficient Matching," Economic Theory, Vol. 30, 243-263.

[23] Edelman, B. G. (2015): "How to Launch Your Digital Platform: A Playbook for Strategists." Harvard Business Review 93(4), 90-97.

[24] Evans, D. and R. Schmalensee (2015): "Matchmakers: The New Economics of Multisided Platforms," Harvard Business Review Press.

[25] Farrell, J. and P. Klemperer (2006): "Coordination and Lock-In: Competition with Switching Costs and Network Effects," in Armstrong, M. and Porter, R. (eds) Handbook of Industrial Organization, Vol. 3 (North-Holland), 1967-2072. 
[26] Gallaway, B. (2013): "Confusion Ruled the Day at Microsoft's Xbox One Reveal Event," https://www.wired.com/2013/2005/xbox-one-hands-on/.

[27] Gaudeul, A. (2010): "Software Marketing on the Internet: the Use of Samples and Repositories," Economics of Innovation and New Technology, 19(3), 259-281.

[28] Godes, D., Mayzlin, D., Chen, Y., Das, S., Dellarocas, C., Pfeiffer, B., Libai, B., Sen, S., Shi, M. and P. Verlegh (2005): "The Firm's Management of Social Interactions," Marketing Letters, 16(3-4), pp.415-428.

[29] Gomes, R. and A. Pavan (2016): "Many-To-Many Matching and Price Discrimination," Theoretical Economics, 11, 1005-1052.

[30] Gomes, R. and A. Pavan (2017): "Price Customization and Targeting in Many-to-Many matching markets," mimeo Northwestern University and Toulouse School of Economics.

[31] Halaburda, H. and Y. Yehezkel (2013): "Platform Competition under Asymmetric Information," American Economic Journal: Microeconomics, 5(3): 22-68.

[32] Hagiu, A. (2006): "Optimal Pricing and Commitment in Two-Sided Markets," The RAND Journal of Economics 37(3), 720-737.

[33] Hagiu, A. (2009): "Two-Sided Platforms: Product Variety and Pricing Structures," Journal of Economics and Management Strategy 18, 1011-1043.

[34] Hagiu, A. and H. Halaburda (2014): "Information and Two-sided Platform Profits," International Journal of Industrial Organization, 34(C), 25-35.

[35] Haruvy, E. and A. Prasad (2005): "Freeware as a Competitive Deterrent," Information Economics and Policy, 17(4), pp.513-534.

[36] Johnson, J. and D. Myatt (2006): "On the Simple Economics of Advertising, Marketing, and Product Design" American Economic Review 96,(3), 756-784

[37] Jullien, B. (2007): "Two-Sided Markets and Electronic Intermediation," in G. Illing and M. Peitz (eds.), Industrial Organization and the Digital Economy, MIT Press.

[38] Katz M., and C.Shapiro (1985): "Network Externalities, Competition and Compatibility," American Economic Review, 75, 424-440.

[39] Lee, R. S. (2013): "Vertical Integration and Exclusivity in Platform and Two-sided Markets," American Economic Review 103(7), 2960-3000.

[40] Lee, Y., and O'Connor, G. C. (2003): "New product launch strategy for network effects products," Journal of the Academy of Marketing Science, 31(3), 241-255. 
[41] Lewis, T. and D. E. M. Sappington (1994): "Supplying Information to Facilitate Price Discrimination," International Economic Review 35, 309-327.

[42] Mitchell, M. and A. Skrzypacz (2006): "Network Externalities and Long-Run Market Share," Economic Theory, 29, 621-648.

[43] Morris, S. and H.S. Shin (2003): "Global Games - Theory and Applications," in Advances in Economics and Econometrics, 8th World Congress of the Econometric Society (M. Dewatripont, L. Hansen, and S. Turnovsky, eds.), Cambridge University Press.

[44] Pisano, G. P. and R. Verganti (2008), "Which Kind of Collaboration is Right for You," Harvard Business Review, 86(12), 78-86.

[45] Reillier, L. C., and Reillier, B. (2017). Platform Strategy: How to Unlock the Power of Communities and Networks to Grow Your Business. Taylor \& Francis.

[46] Rochet, J.C. and J. Tirole (2003): "Platform Competition in Two-Sided Markets," Journal of the European Economic Association 1, 990-1029.

[47] Rochet, J.C. and J. Tirole (2006): "Two-Sided Markets: a Progress Report," The RAND Journal of Economics 37, 645-667.

[48] Rysman, M. (2009): "The Economics of Two-Sided Markets," Journal of Economic Perspective $23(3), 125-143$.

[49] Sheshinski, E.(1976: "Price, Quality and Quantity Regulation in Monopoly Situations," Economica, London School of Economics and Political Science, vol. 43(17), 127-37.

[50] Spence, A.M. (1975): "Monopoly, Quality, and Regulation," Bell Journal of Economics, 6(2), $417-29$.

[51] Sterlicchi, J. (2013): "Nintendo's Wii console captures new game market," https://www.theguardian.com/business/2007/oct/2010/usnews.internationalnews.

[52] Tan, G. and J. Zhou (2017): "Price Competition in Multi-Sided Markets," mimeo University of Southern California and National University of Singapore.

[53] Veiga, A. White, A. and E. G. Weyl (2017): "Multidimensional Platform Design," American Economic Review Papers and Proceedings, 107(5).

[54] Weyl, E. G. (2010): "A Price Theory of Multi-sided Platforms," American Economic Review, 100(4): 1642-72.

[55] Whinston, M. (1990): "Tying, Foreclosure, and Exclusion," American Economic Review 80(4), 837-859. 
[56] White, A. and E. G. Weyl (2017) "Insulated Platform Competition", mimeo Microsoft Research.

\section{Appendix}

\section{Benchmarks and monopoly}

Proof of Proposition 1. Under the profit-maximizing prices, the expected participation rates $Q_{i}^{k}$, $i=1,2, k=A, B$, must satisfy the familiar first-order conditions

$$
p_{i}^{k}+\left.Q_{i} \frac{\partial p_{i}^{k}}{\partial Q_{i}}\right|_{p_{i}^{-k}=\text { const }}=0 .
$$

Combining the above optimality conditions with the fact that, given any vector of prices, the demands are given by $Q_{i}^{k}\left(\hat{v}_{i}\right), i=1,2, k=A, B$, with $\hat{v}_{i}=p_{i}^{B}-p_{i}^{A}$ then gives the result. Q.E.D.

Lemma 1. Suppose information is complete and $d^{35}$

$$
\gamma_{1} \gamma_{2}<\frac{1}{4 \max _{x} \lambda_{1}^{\theta}(x) \max _{x} \lambda_{2}^{\theta}(x)}
$$

For any vector of prices $p=\left(p_{1}^{A}, p_{2}^{A}, p_{1}^{B}, p_{2}^{B}\right)$, there exists one and only one continuation equilibrium.

Proof of Lemma 1. We establish the result by showing that, for any $p=\left(p_{1}^{A}, p_{2}^{A}, p_{1}^{B}, p_{2}^{B}\right)$, there exists one, and only one, solution to the system of equations given by (2). This in turn implies existence and uniqueness of continuation equilibria in monotone strategies. The result in the lemma then follows from the fact that the unique monotone continuation equilibrium is also the unique continuation strategy profile surviving iterated deletion of interim strictly dominated strategies (that is, it is the unique rationalizable profile).

Fix the prices $p=\left(p_{1}^{A}, p_{2}^{A}, p_{1}^{B}, p_{2}^{B}\right) \in \mathbb{R}^{4}$ and observe that, under complete information, Condition (2) is equivalent to $\hat{v}_{i}+\gamma_{i}\left[1-2 \Lambda_{j}^{\theta}\left(\hat{v}_{j}\right)\right]=p_{i}^{B}-p_{i}^{A}, i, j=1,2, j \neq i$.

For any $\hat{v}_{j} \in \mathbb{R}, j=1,2$, the gross payoff differential that an agent from side $i \neq j$ with differential in stand-alone valuations $v_{i}$ derives from joining platform $B$ relative to joining platform $A$, when all agents from side $j$ follow a cut-off strategy with cutoff $\hat{v}_{j}^{36}$ is strictly increasing in $v_{i}$ and equal to $v_{i}+\gamma_{i}\left[1-2 \Lambda_{j}^{\theta}\left(\hat{v}_{j}\right)\right]$. Note that the term $1-2 \Lambda_{j}^{\theta}\left(\hat{v}_{j}\right)=q_{j}^{B}-q_{j}^{A}$ is the differential between the side- $j$ participation to platform $B$ and the side- $j$ participation to platform $A$. This means that, given any $\hat{v}_{j}$, there exists one and only one solution $\hat{v}_{i}=r_{i}\left(\hat{v}_{j}\right)$ to the equation $p_{i}^{B}-p_{i}^{A}=\hat{v}_{i}+\gamma_{i}\left[1-2 \Lambda_{j}^{\theta}\left(\hat{v}_{j}\right)\right]$.

\footnotetext{
${ }^{35}$ Recall that $\lambda_{i}^{\theta}\left(v_{i}\right)$ is the density of the side- $i$ cumulative marginal distribution $\Lambda_{i}^{\theta}\left(v_{i}\right)$ of stand-alone differentials in state $\theta$.

${ }^{36}$ That is, join platform $A$ when $v_{j l}<\hat{v}_{j}$ and join platform $B$ when $v_{j l}>\hat{v}_{j}$.
} 
Now consider the function $y\left(v_{j}\right) \equiv v_{j}+\gamma_{j}\left[1-2 \Lambda_{i}^{\theta}\left(r_{i}\left(v_{j}\right)\right)\right]$. Note that $y\left(v_{j}\right)$ is the gross payoff differential between joining platform $B$ and joining platform $A$ for an agent from side $j$ with differential in stand-alone valuations equal to $v_{j}$, when all agents from side $i \neq j$ follow a cut-off strategy with cut-off equal to $r_{i}\left(v_{j}\right)$. The function $y\left(v_{j}\right)$ is differentiable with derivative

$$
y^{\prime}\left(v_{j}\right)=1-2 \gamma_{j} \lambda_{i}^{\theta}\left(r_{i}\left(v_{j}\right)\right) r_{i}^{\prime}\left(v_{j}\right)=1-4 \gamma_{i} \gamma_{j} \lambda_{i}^{\theta}\left(r_{i}\left(v_{j}\right)\right) \lambda_{j}^{\theta}\left(v_{j}\right)
$$

Condition (27) guarantees that $y^{\prime}\left(v_{j}\right)>0$ for all $v_{j} \in \mathbb{R}$. Because $\lim _{v_{j} \rightarrow-\infty} y\left(v_{j}\right)=-\infty$ and $\lim _{v_{j} \rightarrow+\infty} y\left(v_{j}\right)=+\infty$, we then have that a solution to the equation $y\left(v_{j}\right)=p_{j}^{B}-p_{j}^{A}$ exists and is unique. In turn, this implies existence and uniqueness of a solution to the system of equations given by (2). Q.E.D.

Proof of Proposition 2. For any vector of participation rates $\left(Q_{1}^{B}, Q_{2}^{B}\right)$, the prices that platform $B$ must set to implement the participation rates $Q_{1}^{B}$ and $Q_{2}^{B}$ are given by

$$
p_{i}^{B}=p_{i}^{A}+\hat{v}_{i}+2 \gamma_{i} Q_{j}^{B}-\gamma_{i}, i, j=1,2, j \neq i,
$$

with $\hat{v}_{1}$ and $\hat{v}_{1}$ implicitly defined by $Q_{i}^{B}\left(\hat{v}_{i}\right)=Q_{i}^{B}, i=1,2$. Platform $B$ 's profits (expressed as a function of the participation rates $\left.\left(Q_{1}^{B}, Q_{2}^{B}\right)\right)$ are thus equal to

$$
\Pi^{B}=\sum_{i, j=1,2, j \neq i}\left[p_{i}^{A}+\hat{v}_{i}+2 \gamma_{i} Q_{j}^{B}-\gamma_{i}\right] Q_{i}^{B} .
$$

The participation rates that maximize platform $B$ 's profits must thus solve the following first-order conditions

$$
p_{i}^{B}-\frac{Q_{i}^{B}}{\left|d Q_{i}^{B}\left(\hat{v}_{i}\right) / d v_{i}\right|}+2 \gamma_{j} Q_{j}^{B}=0
$$

Similar conditions apply to platform $A$. Expressing the optimality conditions in (28) in terms of prices and participation thresholds then permits us to establish the result. Q.E.D.

Proof of Proposition 3. The result follows from differentiating the profit function in (10) and then using (8). Q.E.D.

\section{Duopoly analysis}

Lemma 2. Suppose information is dispersed and Conditions (M) and (Q) hold. For any vector of prices $p=\left(p_{1}^{A}, p_{2}^{A}, p_{1}^{B}, p_{2}^{B}\right)$, there exists one and only one solution to the system of equations given by (14). In every continuation equilibrium, all agents from side $i=1,2$ whose differential in stand-alone valuations exceeds $\hat{v}_{i}$ join platform $B$, whereas all agents whose differential in stand-alone valuation is less than $\hat{v}_{i}$ join platform $A$, where $\left(\hat{v}_{1}, \hat{v}_{2}\right)$ is the unique solution to (14).

Proof of Lemma 2. The proof parallels that for Lemma 1 above. First observe that, when Condition (M) holds, for any $v_{j} \in \mathbb{R}$, the gross payoff differential that an agent from side $i \neq j$ with 
differential in stand-alone valuations equal to $v_{i}$ derives from joining platform $B$ relative to joining platform $A$, when all agents from side $j$ follow a cut-off strategy with cutoff $v_{j}$ is strictly increasing in $v_{i}$ and equal to $v_{i}+\gamma_{i}\left[1-2 M_{j i}\left(v_{j} \mid v_{i}\right)\right]$. The intermediate-value and implicit-function theorems then imply that, given the prices $p=\left(p_{1}^{A}, p_{2}^{A}, p_{1}^{B}, p_{2}^{B}\right)$, for any $v_{j} \in \mathbb{R}$, there exists one and only one solution $v_{i}=\zeta_{i}\left(v_{j}\right)$ to the equation $p_{i}^{B}-p_{i}^{A}=v_{i}+\gamma_{i}\left[1-2 M_{j i}\left(v_{j} \mid v_{i}\right)\right]$ with $\zeta_{i}\left(v_{j}\right)$ satisfying

$$
\zeta_{i}^{\prime}\left(v_{j}\right)=\frac{2 \gamma_{i} \frac{\partial M_{j i}\left(v_{j} \mid \zeta_{i}\left(v_{j}\right)\right)}{\partial v_{j}}}{1-2 \gamma_{i} \frac{\partial M_{j i}\left(v_{j} \mid \zeta_{i}\left(v_{j}\right)\right)}{\partial v_{i}}} .
$$

Note that the denominator in (29) is strictly positive under Condition (M) while the nominator is also positive given that $\partial M_{j i}\left(v_{j} \mid \zeta_{i}\left(v_{j}\right)\right) / \partial v_{j}$ is simply the density of the posterior beliefs of any agent from side $i$ with stand-alone differential equal to $\zeta_{i}\left(v_{j}\right)$.

Next, let $z_{j}\left(v_{j}\right) \equiv v_{j}+\gamma_{j}\left[1-2 M_{i j}\left(\zeta_{i}\left(v_{j}\right) \mid v_{j}\right)\right]$ denote the gross payoff differential between joining platform $B$ and joining platform $A$ for an agent from side $j$ with differential in stand-alone valuations equal to $v_{j}$, when all agents from side $i \neq j$ follow a cut-off strategy with cut-off equal to $\zeta_{i}\left(v_{j}\right)$. The function $z_{j}\left(v_{j}\right)$ is differentiable with derivative equal to

$$
\begin{aligned}
z_{j}^{\prime}\left(v_{j}\right) & =1-2 \gamma_{j}\left\{\frac{\partial M_{i j}\left(\zeta_{i}\left(v_{j}\right) \mid v_{j}\right)}{\partial v_{i}} \zeta_{i}^{\prime}\left(v_{j}\right)+\frac{\partial M_{i j}\left(\zeta_{i}\left(v_{j}\right) \mid v_{j}\right)}{\partial v_{j}}\right\} \\
& =1-2 \gamma_{j} \frac{\partial M_{i j}\left(\zeta_{i}\left(v_{j}\right) \mid v_{j}\right)}{\partial v_{j}}-\left(\frac{4}{1-2 \gamma_{i} \frac{\partial M_{j i}\left(v_{j} \mid \zeta_{i}\left(v_{j}\right)\right)}{\partial v_{i}}}\right) \gamma_{i} \gamma_{j} \frac{\partial M_{i j}\left(\zeta_{i}\left(v_{j}\right) \mid v_{j}\right)}{\partial v_{i}} \frac{\partial M_{j i}\left(v_{j} \mid \zeta_{i}\left(v_{j}\right)\right)}{\partial v_{j}} .
\end{aligned}
$$

Together, Conditions (M) and (Q) imply that the function $z_{j}\left(v_{j}\right)$ is strictly increasing. Because $\lim _{v_{j} \rightarrow-\infty} z_{j}\left(v_{j}\right)=-\infty$ and $\lim _{v_{j} \rightarrow+\infty} z_{j}\left(v_{j}\right)=+\infty$, we then have that a solution to the equation $z_{j}\left(v_{j}\right)=p_{j}^{B}-p_{j}^{A}$ exists and is unique. This in turn implies that there exists one and only one solution to the system of equations given by (14). Q.E.D.

Proof of Proposition 4. Given the prices $\left(p_{1}^{A}, p_{2}^{A}\right)$ set by platform $A$, and given the bijective relationship between $\left(p_{1}^{B}, p_{2}^{B}\right)$ and $\left(\hat{v}_{1}, \hat{v}_{2}\right)$ given by $(14)$, platform $B$ 's prices $\left(p_{1}^{B}, p_{2}^{B}\right)$ constitute a best-response to $\left(p_{1}^{A}, p_{2}^{A}\right)$ if, and only if, the participation thresholds $\left(\hat{v}_{1}, \hat{v}_{2}\right)$ they induce solve the following problem:

$$
\max _{\left(v_{1}, v_{2}\right)} \hat{\Pi}^{B}\left(v_{1}, v_{2}\right) \equiv \sum_{i, j=1,2, j \neq i}\left[p_{i}^{A}+v_{i}+\gamma_{i}-2 \gamma_{i} M_{j i}\left(v_{j} \mid v_{i}\right)\right] Q_{i}^{B}\left(v_{i}\right)
$$

The thresholds $\left(\hat{v}_{1}, \hat{v}_{2}\right)$ that maximize platform $B$ 's profits must thus be a solution to the first-order conditions given by

$$
\begin{gathered}
-\left[p_{i}^{A}+\hat{v}_{i}-2 \gamma_{i} M_{j i}\left(\hat{v}_{j} \mid \hat{v}_{i}\right)+\gamma_{i}\right]\left|\frac{d Q_{i}^{B}\left(\hat{v}_{i}\right)}{d v_{i}}\right|+Q_{i}^{B}\left(v_{i}\right)-2 \gamma_{i} \frac{\partial M_{j i}\left(\hat{v}_{j} \mid \hat{v}_{i}\right)}{\partial v_{i}} Q_{i}^{B}\left(v_{i}\right) \\
-2 \gamma_{j} \frac{\partial M_{i j}\left(\hat{v}_{i} \mid \hat{v}_{j}\right)}{\partial v_{i}} Q_{j}^{B}\left(v_{j}\right)=0 .
\end{gathered}
$$


The result then follows from combining the above optimality conditions with their analogs for platform $A$ and using (14). Q.E.D.

Proof of Corollary 1. When competition is symmetric, the participation thresholds are given by $\hat{v}_{1}=\hat{v}_{2}=0$ (This follows directly from property (b) in the definition of symmetric competition). From (14), and using property (a) in the definition of symmetric competition, we then have that $M_{j i}(0 \mid 0)=1 / 2, i, j=1,2, j \neq i$. That the platforms expect to share the market evenly then implies that $Q_{i}^{k}(0)=1 / 2, k=A, B, i=1,2$. From (15), we then have that the equilibrium prices must satisfy

$$
p_{i}^{k}=\frac{1}{2\left|d Q_{i}^{k}(0) / d v_{i}\right|}-\gamma_{i}\left[\frac{\frac{\partial M_{j i}(0 \mid 0)}{\partial v_{i}}}{\left|d Q_{i}^{k}(0) / d v_{i}\right|}\right]-\gamma_{j}\left[\frac{\frac{\partial M_{i j}(0 \mid 0)}{\partial v_{i}}}{\left|d Q_{i}^{k}(0) / d v_{i}\right|}\right], k=A, B .
$$

For the platforms' prices to coincide, it must then be that

$$
\begin{aligned}
& \frac{1}{\left|d Q_{i}^{A}(0) / d v_{i}\right|}\left(\frac{1}{2}-\gamma_{i} \frac{\partial M_{j i}(0 \mid 0)}{\partial v_{i}}-\gamma_{j} \frac{\partial M_{i j}(0 \mid 0)}{\partial v_{i}}\right) \\
& =\frac{1}{\left|d Q_{i}^{B}(0) / d v_{i}\right|}\left(\frac{1}{2}-\gamma_{i} \frac{\partial M_{j i}(0 \mid 0)}{\partial v_{i}}-\gamma_{j} \frac{\partial M_{i j}(0 \mid 0)}{\partial v_{i}}\right)
\end{aligned}
$$

Clearly, it cannot be that

$$
\frac{1}{2}-\gamma_{i} \frac{\partial M_{j i}(0 \mid 0)}{\partial v_{i}}-\gamma_{j} \frac{\partial M_{i j}(0 \mid 0)}{\partial v_{i}}=0
$$

for otherwise the equilibrium prices would be equal to zero on each side, and this is obviously inconsistent with the optimality of the platforms' strategies. We thus conclude that there must exist $\psi_{i}(0) \in \mathbb{R}, i=1,2$, such that $\left|d Q_{i}^{k}(0) / d v_{i}\right|=\psi_{i}(0), i=1,2, k=A, B$. Together, the above properties imply that the equilibrium prices must satisfy (16). The other statements in the corollary follow directly from the equilibrium price formulas. Q.E.D.

\section{Information management}

Proof of Proposition 5. The formulas for the equilibrium prices in (19) follow directly from Corollary 1 by noting that $\psi_{i}(0)=\sqrt{\beta_{i}^{v}} \phi(0), \partial M_{j i}(0 \mid 0) / \partial v_{i}=-\Omega \sqrt{\beta_{i}^{v}} \phi(0)$, and $\partial M_{i j}(0 \mid 0) / \partial v_{i}=$ $\sqrt{1+\Omega^{2}} \sqrt{\beta_{i}^{v}} \phi(0)$. Q.E.D.

Proof of Proposition 6. We first establish the effect of variations in $\rho_{v}$ on equilibrium profits and then their effect on welfare and consumer surplus.

Equilibrium profits. From (20), equilibrium profits are increasing in the coefficient of mutual forecastability $\Omega$. That equilibrium profits increase with $\rho_{v}$ follows from the fact that $\Omega=\rho_{v} / \sqrt{1-\rho_{v}^{2}}$ is increasing in $\rho_{v}$. 
Total welfare. Let $G_{i}^{\theta}$ denote the joint distribution of $\left(V_{i}, v_{i}\right)$ in state $\theta$, with marginals given by $G_{i V}^{\theta}$ and $G_{i v}^{\theta}, i=1,2$. Recall that the aggregate state is parametrized by a vector $\theta=\left(\theta_{1}, \theta_{2}\right)$ drawn from a distribution $F_{\theta}$. Given $\theta$, each pair $\left(V_{i l}, v_{i l}\right), i=1,2, l \in[0,1]$ is drawn independently across agents from $G_{i}^{\theta}$.

In equilibrium, each agent $l$ from each side $i=1,2$, joins platform $A$ when $v_{i l}<0$ and platform $B$ when $v_{i l}>0$. This means that total ex-ante welfare is equal to $W=\mathbb{E}[w(\theta)]$, where

$$
\begin{aligned}
w(\theta) \equiv & \sum_{i, j=1,2, j \neq i} \int_{v_{i}=-\infty}^{0} \int_{V_{i}=-\infty}^{V_{i}=+\infty}\left\{s_{i}-\frac{V_{i}}{2}+\gamma_{i} q_{j}^{A}(\theta)\right\} d G_{i}^{\theta}\left(V_{i}, v_{i}\right) \\
& +\sum_{i, j=1,2, j \neq i} \int_{v_{i}=0}^{+\infty} \int_{V_{i}=-\infty}^{V_{i}=+\infty}\left\{s_{i}+\frac{V_{i}}{2}+\gamma_{i} q_{j}^{B}(\theta)\right\} d G_{i}^{\theta}\left(V_{i}, v_{i}\right),
\end{aligned}
$$

with

$$
q_{j}^{A}(\theta)=G_{j x}^{\theta}(0)=1-q_{j}^{B}(\theta)
$$

denoting the measure of agents from side $j$ who join platform $A$.

Now observe that

$$
\begin{aligned}
w(\theta)= & \sum_{i=1,2} \int_{V_{i}=-\infty}^{V_{i}=+\infty}\left\{s_{i}-\frac{V_{i}}{2}\right\} d G_{i V}^{\theta}\left(V_{i}\right)+\sum_{i=1,2} \int_{v_{i}=0}^{+\infty} \int_{V_{i}=-\infty}^{V_{i}=+\infty} V_{i} d G_{i}^{\theta}\left(V_{i}, v_{i}\right) \\
& +\sum_{i, j=1,2} \gamma_{i} G_{i v}^{\theta}(0) G_{j v}^{\theta}(0)+\sum_{i, j=1,2} \gamma_{i}\left[1-G_{i v}^{\theta}(0)\right]\left[1-G_{j v}^{\theta}(0)\right] .
\end{aligned}
$$

Averaging over $\theta$, and using the law of iterated expectations, we then have that

$$
W=K+\sum_{i=1,2} \mathbb{E}\left[V_{i l} \mathbb{I}\left(v_{i l}>0\right)\right]+\left(\gamma_{1}+\gamma_{2}\right) \operatorname{Pr}\left(v_{1} \cdot v_{2} \geq 0\right)
$$

where

$$
K=\sum_{i=1,2} \mathbb{E}\left[s_{i}-\frac{V_{i}}{2}\right]
$$

In the Gaussian case,

$$
\operatorname{Pr}\left(v_{1} \geq 0, v_{2} \geq 0\right)=\operatorname{Pr}\left(v_{1}<0, v_{2}<0\right)=\frac{1}{4}+\frac{1}{2 \pi} \arcsin \left(\rho_{v}\right)=\frac{1}{4}+\phi^{2}(0) \arcsin \left(\rho_{v}\right)
$$

and hence

$$
\operatorname{Pr}\left(v_{1} \cdot v_{2} \geq 0\right)=\frac{1}{2}+2 \phi^{2}(0) \arcsin \left(\rho_{v}\right)
$$

Also observe that

$$
\frac{\partial \arcsin \left(\rho_{v}\right)}{\partial \rho_{v}}=\frac{1}{\sqrt{1-\rho_{v}^{2}}}=\frac{\Omega}{\rho_{v}} \geq 0 .
$$

The first two terms in (32) are invariant in $\rho_{v}$. Hence, total welfare also increases with $\rho_{v}$. 
Consumer surplus. Finally, consider the effects of variations in $\rho_{v}$ on consumer surplus. Observe that $C S=W-2 \Pi^{*}$. Using the fact that

$$
\Omega-\sqrt{1+\Omega^{2}}=\frac{\rho_{v}-1}{\sqrt{1-\rho_{v}^{2}}}
$$

we have that

$$
\frac{\partial\left(2 \Pi^{*}\right)}{\partial \rho_{v}}=\left(\gamma_{1}+\gamma_{2}\right) \frac{\partial}{\partial \rho_{v}}\left(\frac{\rho_{v}-1}{\sqrt{1-\rho_{v}^{2}}}\right)
$$

and hence that

$$
\frac{\partial C S}{\partial \rho_{v}}=\frac{\partial W}{\partial \rho_{v}}-\frac{\partial\left(2 \Pi^{*}\right)}{\partial \rho_{v}}=2\left(\gamma_{1}+\gamma_{2}\right) \phi^{2}(0) \frac{1}{\sqrt{1-\rho_{v}^{2}}}-\left(\gamma_{1}+\gamma_{2}\right) \frac{\partial}{\partial \rho_{v}}\left(\frac{\rho_{v}-1}{\sqrt{1-\rho_{v}^{2}}}\right)
$$

It follows that

$$
\frac{\partial C S}{\partial \rho_{v}}=\frac{\left(\gamma_{1}+\gamma_{2}\right)}{\sqrt{1-\rho_{v}^{2}}}\left\{2 \phi^{2}(0)-\frac{1}{1+\rho_{v}}\right\} .
$$

Because $2 \phi^{2}(0)=1 / \pi$ and $\pi>1+\rho_{v}$, we conclude that CS is decreasing in $\rho_{v}$. Q.E.D.

Proof of Proposition 7. We establish the result by considering separately the effects of such policies on equilibrium profits, welfare, and consumer surplus.

Profits. Recall that equilibrium profits are increasing in $\Omega$. When preferences are aligned (i.e., $\left.\rho_{v}>0\right)$, an increase in the agents' ability to predict participation decisions on the opposite side (formally, an increase in $|\Omega|$ ) then implies an increase in $\Omega$ and hence higher profits. When, instead, preferences are misaligned (i.e., $\rho_{v}<0$ ), an increase in $|\Omega|$ implies a reduction of $\Omega$ and hence lower profits.

Welfare. Next, consider the effects of such policies on total welfare. Recall that the latter is given by the formula in (32). Note that policies that leave unchanged both the prior distribution from which the true stand-alone valuations $V_{i}$ are drawn and the ex-ante distribution of the estimated stand-alone differentials $v_{i}$ also leave the first two terms in (32) unchanged. To see this, observe that $\operatorname{Cov}\left(V_{i}, v_{i}\right)=1 / \beta_{i}^{v}$ (to see this, just note that $V_{i}=v_{i}+$ error where, given $v_{i}$, error is orthogonal to $\left.V_{i}\right)$. Hence the joint distribution of $\left(V_{i}, v_{i}\right)$ is unaffected by such variations and hence so is

$$
\sum_{i=1,2} \mathbb{E}\left[s_{i}-\frac{V_{i}}{2}\right]+\sum_{i=1,2} \mathbb{E}\left[V_{i l} \mathbb{I}\left(v_{i l}>0\right)\right]
$$

From (32), it is then immediate that policies that increase the agents' ability to predict participation decisions on the other side of the market increase welfare if, and, only if, they increase $\arcsin \left(\rho_{v}\right)$. Then use (33) to observe that $\arcsin \left(\rho_{v}\right)$ is increasing in $\rho_{v}$, and hence in $\Omega$. The effects of such policies on welfare are thus the same as the effects of the same policies on equilibrium profits.

Consumer surplus. Lastly, consider the effects of such policies on consumer surplus. From the same derivations as in the proof of Proposition 6,

$$
\frac{\partial C S}{\partial \rho_{v}}=\frac{\left(\gamma_{1}+\gamma_{2}\right)}{\sqrt{1-\rho_{v}^{2}}}\left\{\frac{1}{\pi}-\frac{1}{1+\rho_{v}}\right\}<0 .
$$


Hence, the effects of such policies on consumer surplus are of opposite sign than the effects of such policies on profits and total welfare. Q.E.D.

Proof of Proposition 8. The proof is in three parts. Part 1 establishes that the equilibrium prices must satisfy the optimality conditions in (24). Part 2 establishes the comparisons of equilibrium prices across the two environments considered in the proposition. Part 3 shows how the conditions in the proposition specialize in the Gaussian model.

Part 1. Fix the prices $\left(p_{1}^{B}, p_{2}^{B}\right)$ set by platform $B$. Platform $A$ 's expected profits, expressed as a function of the period-1 participation thresholds they induce, are given by

$$
\Pi^{A}\left(\hat{v}_{1}, \hat{v}_{2}\right)=\sum_{i, j=1,2, j \neq i}\left[p_{i}^{B}-\hat{v}_{i}+2 \gamma_{i} M_{j i}\left(\hat{v}_{j} \mid \hat{v}_{i}\right)-\gamma_{i}\right] \mathbf{Q}_{i}^{A}\left(\hat{v}_{1}, \hat{v}_{2}\right) .
$$

The best response by platform $A$ to platform $B$ 's prices $\left(p_{1}^{B}, p_{2}^{B}\right)$ then consists in inducing period-1 thresholds $\left(\hat{v}_{1}, \hat{v}_{2}\right)$ that solve the following optimality conditions

$$
\begin{aligned}
& {\left[p_{i}^{B}-\hat{v}_{i}+2 \gamma_{i} M_{j i}\left(\hat{v}_{j} \mid \hat{v}_{i}\right)-\gamma_{i}\right] \frac{\partial \mathbf{Q}_{i}^{A}\left(\hat{v}_{1}, \hat{v}_{2}\right)}{\partial v_{i}}-\left[1-2 \gamma_{i} \frac{\partial M_{j i}\left(\hat{v}_{j} \mid \hat{v}_{i}\right)}{\partial v_{i}}\right] \mathbf{Q}_{i}^{A}\left(\hat{v}_{1}, \hat{v}_{2}\right)} \\
& +2 \gamma_{j} \frac{\partial M_{i j}\left(\hat{v}_{i} \mid \hat{v}_{j}\right)}{\partial v_{i}} \mathbf{Q}_{j}^{A}\left(\hat{v}_{1}, \hat{v}_{2}\right)+\left[p_{j}^{B}-\hat{v}_{j}+2 \gamma_{j} M_{i j}\left(\hat{v}_{i} \mid \hat{v}_{j}\right)-\gamma_{j}\right] \frac{\partial \mathbf{Q}_{j}^{A}\left(\hat{v}_{1}, \hat{v}_{2}\right)}{\partial v_{i}}=0 .
\end{aligned}
$$

Replacing $p_{i}^{A}=p_{i}^{B}-\hat{v}_{i}+2 \gamma_{i} M_{j i}\left(\hat{v}_{j} \mid \hat{v}_{i}\right)-\gamma_{i}$ and $p_{j}^{A}=p_{j}^{B}-\hat{v}_{j}+2 \gamma_{j} M_{i j}\left(\hat{v}_{i} \mid \hat{v}_{j}\right)-\gamma_{j}$ into the above optimality conditions and rearranging terms, we obtain (24). Clearly, similar conditions hold for platform $B$.

Part 2. When competition is symmetric, the equilibrium prices under post-launch disclosures are given by

$$
p_{i}^{* *}=\frac{1}{\partial \mathbf{Q}_{i}^{A}(0,0) / \partial v_{i}}\left\{1-2 \gamma_{j} \frac{\partial M_{i j}(0 \mid 0)}{\partial v_{i}}-2 \gamma_{i} \frac{\partial M_{j i}(0 \mid 0)}{\partial v_{i}}\right\}-p_{j}^{* *} \frac{\partial \mathbf{Q}_{j}^{A}(0,0) / \partial v_{i}}{\partial \mathbf{Q}_{i}^{A}(0,0) / \partial v_{i}}
$$

$i, j=1,2, j \neq i$, where

$$
\partial \mathbf{Q}_{i}^{A}(0,0) / \partial v_{i}=\mathbb{E}_{\theta}\left[\lambda_{i}^{\theta}(0)+\lambda_{i}^{\theta}\left(v_{i}^{\theta}(0,0)\right) \frac{\partial v_{i}^{\theta}(0,0)}{\partial v_{i}}\right]
$$

and

$$
\partial \mathbf{Q}_{j}^{A}(0,0) / \partial v_{i}=\mathbb{E}_{\theta}\left[\lambda_{j}^{\theta}\left(v_{j}^{\theta}(0,0)\right) \frac{\partial v_{j}^{\theta}(0,0)}{\partial v_{i}}\right] .
$$

In the absence of disclosure, instead, the equilibrium prices are given by

$$
p_{i}^{*}=\frac{1}{2\left[\partial Q_{i}^{A}(0) / \partial v_{i}\right]}\left\{1-2 \gamma_{j} \frac{\partial M_{i j}(0 \mid 0)}{\partial v_{i}}-2 \gamma_{i} \frac{\partial M_{j i}(0 \mid 0)}{\partial v_{i}}\right\}
$$

$i=1,2$, where $\partial Q_{i}^{A}(0) / \partial v_{i}=\psi_{i}(0)=\mathbb{E}_{\theta}\left[\lambda_{i}^{\theta}(0)\right]$. 
Because the expected intertemporal demands are the same with and without post-launch disclosures, equilibrium profits are higher (alternatively, lower) with post-launch disclosures if $p_{1}^{* *}+p_{2}^{* *} \geq$ $p_{1}^{*}+p_{2}^{*}$ (alternatively, if $\left.p_{1}^{* *}+p_{2}^{* *} \leq p_{1}^{*}+p_{2}^{*}\right)$.

From (34) and (37), it is then evident that, when, in the absence of post-launch disclosures, platforms set positive prices on both sides of the market (i.e., when $p_{1}^{*}, p_{2}^{*} \geq 0$ ), equilibrium prices on both sides are higher under post-launch disclosures than in their absence when, for $i, j=1,2, j \neq i$,

$$
\partial \mathbf{Q}_{i}^{A}(0,0) / \partial v_{i} \leq 2\left[\partial Q_{i}^{A}(0) / \partial v_{i}\right]
$$

and $\partial \mathbf{Q}_{j}^{A}(0,0) / \partial v_{i} \leq 0$, or, equivalently, when

$$
d_{i} \equiv \mathbb{E}_{\theta}\left[\lambda_{i}^{\theta}\left(v_{i}^{\theta}(0,0)\right) \frac{\partial v_{i}^{\theta}(0,0)}{\partial v_{i}}\right]-\mathbb{E}_{\theta}\left[\lambda_{i}^{\theta}(0)\right] \leq 0
$$

and $c_{j i} \equiv \mathbb{E}_{\theta}\left[\lambda_{j}^{\theta}\left(v_{j}^{\theta}(0,0)\right) \frac{\partial v_{j}^{\theta}(0,0)}{\partial v_{i}}\right] \leq 0$ (strictly, when one of the above two inequalities is strict). Symmetrically, when $p_{1}^{*}, p_{2}^{*} \geq 0$, equilibrium prices on both sides of the market are lower under post-launch disclosures if, for $i, j=1,2, j \neq i, d_{i} \geq 0$ and $c_{j i} \geq 0$.

Part 3. To show how the sufficient conditions in the proposition specialize in the Gaussian model, we first need to express the thresholds $v_{i}^{\theta}\left(\hat{v}_{1}, \hat{v}_{2}\right)$ as a function of the primitive parameters. To do this, recall that, for any $\theta$, any pair of period-1 thresholds $\left(\hat{v}_{1}, \hat{v}_{2}\right)$, the associated period-2 thresholds are given by

$$
v_{i}^{\theta}\left(\hat{v}_{1}, \hat{v}_{2}\right)=\hat{v}_{i}-2 \gamma_{i}\left[M_{j i}\left(\hat{v}_{j} \mid \hat{v}_{i}\right)-\Lambda_{j}^{\theta}\left(v_{j}^{\theta}\left(\hat{v}_{1}, \hat{v}_{2}\right)\right)\right], \quad i, j=1,2, j \neq i .
$$

We thus have that

$$
\frac{\partial v_{i}^{\theta}}{\partial v_{i}}=1-2 \gamma_{i} \frac{\partial}{\partial v_{i}} M_{j i}\left(\hat{v}_{j} \mid \hat{v}_{i}\right)+2 \gamma_{i} \lambda_{j}^{\theta}\left(v_{j}^{\theta}\right) \frac{\partial v_{j}^{\theta}}{\partial v_{i}}
$$

and

$$
\frac{\partial v_{j}^{\theta}}{\partial v_{i}}=-2 \gamma_{j} \frac{\partial}{\partial v_{i}} M_{i j}\left(\hat{v}_{i} \mid \hat{v}_{j}\right)+2 \gamma_{j} \lambda_{i}^{\theta}\left(v_{i}^{\theta}\right) \frac{\partial v_{i}^{\theta}}{\partial v_{i}},
$$

where we dropped $\left(\hat{v}_{1}, \hat{v}_{2}\right)$ from the arguments of the period-2 threshold functions $v_{i}^{\theta}$ and $v_{j}^{\theta}$ to ease the notation. From the above two conditions, we obtain that

$$
\frac{\partial v_{i}^{\theta}}{\partial v_{i}}=\frac{1-2 \gamma_{i} \frac{\partial}{\partial v_{i}} M_{j i}\left(\hat{v}_{j} \mid \hat{v}_{i}\right)-4 \gamma_{1} \gamma_{2} \lambda_{j}^{\theta}\left(v_{j}^{\theta}\right) \frac{\partial}{\partial v_{i}} M_{i j}\left(\hat{v}_{i} \mid \hat{v}_{j}\right)}{1-4 \gamma_{1} \gamma_{2} \lambda_{1}^{\theta}\left(v_{1}^{\theta}\right) \lambda_{2}^{\theta}\left(v_{2}^{\theta}\right)}
$$

and

$$
\frac{\partial v_{j}^{\theta}}{\partial v_{i}}=\frac{2 \gamma_{j} \lambda_{i}^{\theta}\left(v_{i}^{\theta}\right)\left(1-2 \gamma_{i} \frac{\partial}{\partial v_{i}} M_{j i}\left(\hat{v}_{j} \mid \hat{v}_{i}\right)\right)-2 \gamma_{j} \frac{\partial}{\partial v_{i}} M_{i j}\left(\hat{v}_{i} \mid \hat{v}_{j}\right)}{1-4 \gamma_{1} \gamma_{2} \lambda_{1}^{\theta}\left(v_{1}^{\theta}\right) \lambda_{2}^{\theta}\left(v_{2}^{\theta}\right)} .
$$

Replacing the above derivatives into the formulas for $d_{i}$ and $c_{j i}$, we obtain that

$$
\begin{aligned}
& d_{i}=\left(1-2 \gamma_{i} \frac{\partial}{\partial v_{i}} M_{j i}(0 \mid 0)\right) \mathbb{E}_{\theta}\left[\frac{\lambda_{i}^{\theta}\left(v_{i}^{\theta}\right)}{1-4 \gamma_{1} \gamma_{2} \lambda_{1}^{\theta}\left(v_{1}^{\theta}\right) \lambda_{2}^{\theta}\left(v_{2}^{\theta}\right)}\right] \\
& -4 \gamma_{1} \gamma_{2} \frac{\partial}{\partial v_{i}} M_{i j}(0 \mid 0) \mathbb{E}_{\theta}\left[\frac{\lambda_{1}^{\theta}\left(v_{1}^{\theta}\right) \lambda_{2}^{\theta}\left(v_{2}^{\theta}\right)}{1-4 \gamma_{1} \gamma_{2} \lambda_{1}^{\theta}\left(v_{1}^{\theta}\right) \lambda_{2}^{\theta}\left(v_{2}^{\theta}\right)}\right]-\psi_{i}(0)
\end{aligned}
$$


and

$$
\begin{aligned}
c_{j i}= & 2 \gamma_{j}\left(1-2 \gamma_{i} \frac{\partial}{\partial v_{i}} M_{j i}(0 \mid 0)\right) \mathbb{E}_{\theta}\left[\frac{\lambda_{1}^{\theta}\left(v_{1}^{\theta}\right) \lambda_{2}^{\theta}\left(v_{2}^{\theta}\right)}{1-4 \gamma_{1} \gamma_{2} \lambda_{1}^{\theta}\left(v_{1}^{\theta}\right) \lambda_{2}^{\theta}\left(v_{2}^{\theta}\right)}\right] \\
& -2 \gamma_{j} \frac{\partial}{\partial v_{i}} M_{i j}(0 \mid 0) \mathbb{E}_{\theta}\left[\frac{\lambda_{j}^{\theta}\left(v_{j}^{\theta}\right)}{1-4 \gamma_{1} \gamma_{2} \lambda_{1}^{\theta}\left(v_{1}^{\theta}\right) \lambda_{2}^{\theta}\left(v_{2}^{\theta}\right)}\right] .
\end{aligned}
$$

Now recall that, in the Gaussian case, $\psi_{i}(0)=\sqrt{\beta_{i}^{v}} \phi(0), \partial M_{j i}(0 \mid 0) / \partial v_{i}=-\Omega \sqrt{\beta_{i}^{v}} \phi(0)$, and $\partial M_{i j}(0 \mid 0) / \partial v_{i}=\sqrt{1+\Omega^{2}} \sqrt{\beta_{i}^{v}} \phi(0)$. Replacing these formulas into the expressions for $d_{i}$ and $c_{j i}$ above, we obtain that

$$
\begin{gathered}
d_{i}=\left(1+2 \gamma_{i} \Omega \sqrt{\beta_{i}^{v}} \phi(0)\right) \mathbb{E}_{\theta}\left[\frac{\lambda_{i}^{\theta}\left(v_{i}^{\theta}\right)}{1-4 \gamma_{1} \gamma_{2} \lambda_{1}^{\theta}\left(v_{1}^{\theta}\right) \lambda_{2}^{\theta}\left(v_{2}^{\theta}\right)}\right] \\
-4 \gamma_{1} \gamma_{2} \sqrt{1+\Omega^{2}} \sqrt{\beta_{i}^{v}} \phi(0) \mathbb{E}_{\theta}\left[\frac{\lambda_{1}^{\theta}\left(v_{1}^{\theta}\right) \lambda_{2}^{\theta}\left(v_{2}^{\theta}\right)}{1-4 \gamma_{1} \gamma_{2} \lambda_{1}^{\theta}\left(v_{1}^{\theta}\right) \lambda_{2}^{\theta}\left(v_{2}^{\theta}\right)}\right]-\sqrt{\beta_{i}^{v}} \phi(0)
\end{gathered}
$$

and

$$
\begin{aligned}
c_{j i} & =2 \gamma_{j}\left(1+2 \gamma_{i} \Omega \sqrt{\beta_{i}^{v}} \phi(0)\right) \mathbb{E}_{\theta}\left[\frac{\lambda_{1}^{\theta}\left(v_{1}^{\theta}\right) \lambda_{2}^{\theta}\left(v_{2}^{\theta}\right)}{1-4 \gamma_{1} \gamma_{2} \lambda_{1}^{\theta}\left(v_{1}^{\theta}\right) \lambda_{2}^{\theta}\left(v_{2}^{\theta}\right)}\right] \\
& -2 \gamma_{j} \sqrt{1+\Omega^{2}} \sqrt{\beta_{i}^{v}} \phi(0) \mathbb{E}_{\theta}\left[\frac{\lambda_{j}^{\theta}\left(v_{j}^{\theta}\right)}{1-4 \gamma_{1} \gamma_{2} \lambda_{1}^{\theta}\left(v_{1}^{\theta}\right) \lambda_{2}^{\theta}\left(v_{2}^{\theta}\right)}\right] .
\end{aligned}
$$

Q.E.D. 
Table 1: Notation summary

\begin{tabular}{l|l}
\hline symbol & description \\
\hline$k=A, B$ & platforms \\
$i, j=1,2$ & sides \\
$l \in[0,1]$ & identity of agents on one side \\
$v_{i l}^{k}$ & stand-alone valuation for platform $k$ of agent $l$ from side $i$ \\
$\gamma_{i}$ & intensity of network effects on side $i$ \\
$q_{i}^{k}$ & mass of agents from side $i$ joining platform $k$ \\
$p_{i}^{k}$ & price charged by platform $k$ on side $i$ \\
$v_{i l}$ & $\equiv v_{i l}^{B}-v_{i l}^{A} ;$ differential in stand-alone valuations \\
$Q_{i}^{A}(v)$ & measure of agents from side $i$ with $v_{i l} \leq v$ expected by platform $A$ \\
$Q_{i}^{B}(v)$ & measure of agents from side $i$ with $v_{i l} \geq v$ expected by platform $B$ \\
$M_{j i}\left(v_{j} \mid v_{i}\right)$ & measure of agents from side $j$ with $v_{j l} \leq v_{j}$ as expected by any side- $i$ agent with $v_{i l}=v_{i}$ \\
$\hat{v}_{i}$ & stand-alone value differential of marginal agent from side $i$ \\
\hline
\end{tabular}

Table 2: Common prior

\begin{tabular}{l|l}
\hline symbol & description \\
\hline$\theta$ & aggregate state \\
$F_{\theta}$ & CDF of $\theta$ \\
$\Lambda_{i}^{\theta}$ & CDF of stand-alone differentials on side $i$ in state $\theta$ \\
$\lambda_{i}^{\theta}$ & PDF of stand-alone differentials on side $i$ in state $\theta$ \\
\hline
\end{tabular}

Table 3: Gaussian structure

\begin{tabular}{l|l}
\hline symbol & description \\
\hline$V_{i l} \equiv V_{i l}^{B}-V_{i l}^{A}$ & differential in stand alone valuations \\
$x_{i l}$ & private signal of agent $l$ from side $i$ \\
$v_{i l} \equiv v_{i l}^{B}-v_{i l}^{A}=\mathbb{E}\left[V_{i l} \mid x_{i l}\right]$ & estimated stand-alone differential of agent $l$ from side $i$ \\
$\rho_{v} \equiv \operatorname{corr}\left(v_{i l}, v_{j l^{\prime}}\right)$ & correlation between estimated stand-alone differentials \\
$\Omega \equiv \frac{\rho_{V}}{\sqrt{1-\rho_{V}^{2}}}$ & of any pair of agents from opposite sides \\
\hline
\end{tabular}

